\title{
An Unexpectedly High Number of New Sutorius (Boletaceae) Species From Northern and Northeastern Thailand
}

OPEN ACCESS

Edited by:

Peter Edward Mortimer, Kunming Institute of Botany, Chinese Academy of Sciences, China

Reviewed by:

Samantha Chandranath Karunarathna,

Kunming Institute of Botany, Chinese Academy of Sciences, China

Zheng Wang

Yale University, United States

*Correspondence: Saisamorn Lumyong scboi009@gmail.com

Olivier Raspe

olivier.ras@mfu.ac.th

Specialty section:

This article was submitted to Evolutionary and Genomic Microbiology, a section of the journal

Frontiers in Microbiology

Received: 18 December 2020 Accepted: 08 March 2021

Published: 12 April 2021

Citation:

Vadthanarat S, Halling RE, Amalfi M, Lumyong $S$ and Raspé $O$ (2021) An Unexpectedly High Number of New Sutorius (Boletaceae) Species

From Northern and Northeastern

Thailand.

Front. Microbiol. 12:643505. doi: 10.3389/fmicb.2021.643505

\section{Santhiti Vadthanarat ${ }^{1,2}$, Roy E. Halling ${ }^{3}$, Mario Amalfi ${ }^{4,5}$, Saisamorn Lumyong ${ }^{1,2,6 *}$ and Olivier Raspé ${ }^{7,8 *}$}

\begin{abstract}
${ }^{1}$ Department of Biology, Faculty of Science, Chiang Mai University, Chiang Mai, Thailand, ${ }^{2}$ Research Center of Microbial Diversity and Sustainable Utilization, Faculty of Science, Chiang Mai University, Chiang Mai, Thailand, ${ }^{3}$ Institute of Systematic Botany, New York Botanical Garden, Bronx, NY, United States, ${ }^{4}$ Botanic Garden Meise, Meise, Belgium, ${ }^{5}$ Fédération Wallonie-Bruxelles, Service Général de l'Enseignement Universitaire et de la Recherche Scientifique, Bruxelles, Belgium, ${ }^{6}$ Academy of Science, The Royal Society of Thailand, Bangkok, Thailand, ${ }^{7}$ School of Science, Mae Fah Luang University, Chiang Rai, Thailand, ${ }^{8}$ Center of Excellence in Fungal Research, Mae Fah Luang University, Chiang Rai, Thailand
\end{abstract}

Sutorius is a poroid genus in Boletaceae that typically has chocolate brown to reddish brown or purplish brown basidiomata with a finely scaly stipe and produces a reddish brown spore deposit. During the survey on diversity of boletes in Northern and Northeastern Thailand, several Sutorius collections were obtained. Combined evidence from morphology and phylogenetic analyses of a combined three-gene data set (atp6, tef1 and rpb2) of the Sutorius collections along with selected Boletaceae in the Pulveroboletus group indicated that Thai collections represent seven new Sutorius species. The analyses also indicated that Tylopilus maculatoides belongs in Sutorius. Therefore, the transfer of T. maculatoides to Sutorius is proposed. Full descriptions and illustrations of the seven new species and $S$. maculatoides are presented in this study. With the seven new species and the new combination, eight of the eleven described Sutorius species are known to occur in Northern and Northeastern Thailand, whereas only one species is known from each of two continents, the Americas and Australia.

Keywords: Boletales, eight new taxa, multigene phylogeny, mushrooms, Pulveroboletus group, taxonomy

\section{INTRODUCTION}

Sutorius Halling, Nuhn and N.A. Fechner was established in 2012 to accommodate two bolete species, Boletus eximius Peck [current name $=$ Sutorius eximius (Peck) Halling, Nuhn and Osmundson] and Leccinum australiense Bougher and Thiers [current name $=$ Sutorius australiensis (Bougher and Thiers) Halling and N.A. Fechner] (Halling et al., 2012). Typical characteristics of Sutorius are stipitate-pileate basidiomata with poroid hymenophore, chocolate to reddish brown or purplish brown basidiomata with finely scaly stipe, and a reddish brown spore deposit. The original publication of Sutorius also reported two Sutorius specimens originating from Chiang Mai Province, in Northern Thailand. One of them was morphologically identified as S. eximius, whereas another was phylogenetically recognized as a different species from S. eximius and S. australiensis. However, the latter was not described as a new species at that time (Halling et al., 2012). In 2016, 
$\mathrm{Wu}$ et al., recombined several bolete species (e.g., Boletus brunneissimus W.F. Chiu; B. hainanensis T.H. Li and M. Zang; B. luridiformis Rostk.; B. obscureumbrinus Hongo; B. tomentulosus M. Zang W.P. Liu and M.R. Hu) into the genus Sutorius based on their phylogenetic inferences. They also described several new Sutorius species from China (e.g., S. ferrugineus G. Wu, F. Li and Zhu L. Yang; S. ferrugineus G. Wu, F. Li and Zhu L. Yang; S. rubriporus G. Wu and Zhu L. Yang). Later, Chai et al. (2019) restudied the phylogenetic relationships of several Boletaceae specimens from subtropical and tropical China, reinstated the genus Neoboletus Gelardi, Simonini and Vizzini and recombined all Sutorius species described in Wu et al. (2016) to be into this latter. Chai et al. (2019) also newly described a third species of Sutorius, Sutorius subrufus N.K. Zeng, H. Chai and S. Jiang from Southern China. Moreover, the phylogeny in that study suggested an additional five undescribed Sutorius species clustering in 10 independent linages (three described species included). That inference also indicated the exemplars identified as S. eximius, originating from several different countries (China, Costa Rica, Indonesia, and United States), were in a polytomic lineage (Chai et al., 2019). So far, the distribution of Sutorius has been recorded to occur in several regions including the Americas, Africa, Asia, and Australia (Singer, 1947; Smith and Thiers, 1971; Fulgenzi et al., 2007; Chandrasrikul et al., 2011; Halling et al., 2012; Chai et al., 2019). At present, only three species have been placed in the genus (Halling et al., 2012; Chai et al., 2019).

In Thailand, there were a few reports of Sutorius species, originally identified as Tylopilus eximius (Peck) Singer (current name $=$ S. eximius) (Chantorn et al., 2007; Chandrasrikul et al., 2008; Vadthanarat, 2009; Vadthanarat et al., 2010). However, the identifications in those reports were based on morphological evidence only, and further phylogenetic studies are needed to confirm those identifications. One Asian species of Boletaceae, Tylopilus maculatoides E. Horak, also has similar morphological characters as Sutorius. That name was proposed for Boletus maculatus Corner non-Raddi based on morphological evidence (Horak, 2011). However, the species has not been included in a phylogenetic context until now (see below).

In this study, morphological and phylogenetic analyses of several Sutorius collections, including T. maculatoides collections, inferred seven new Sutorius species among the Thai collections. Moreover, the phylogram also showed that T. maculatoides clustered within the Sutorius clade with high support. Consequently, the new combination Sutorius maculatoides is proposed. Their phylogenetic affinities and reports of other Sutorius species from Thailand or elsewhere are critically discussed.

\section{MATERIALS AND METHODS}

\section{Specimen Collection}

Fresh basidiomata of Sutorius were collected in Chiang Mai Province in the North and Ubon Ratchathani Province, Northeastern Thailand, in 2012 to 2018 . They were photographed in the field and then wrapped in aluminum foil for later description in the laboratory. The specimens were then dried after the morphological description in an electric drier at $45^{\circ} \mathrm{C}-50^{\circ} \mathrm{C}$. Examined specimens were deposited at Department of National Parks, Wildlife and Plant Conservation, Bangkok, Thailand (BKF), Chiang Mai University, Chiang Mai, Thailand (CMUB), and Mae Fah Luang University, Chiang Rai, Thailand (MFLU) with duplicates in Botanic Garden Meise, Belgium (BR).

\section{Morphological Studies}

Macroscopic descriptions were made based on the detailed field notes and photographs of fresh basidiomata. Color codes are based on Kornerup and Wanscher (1978). Macrochemical reactions (color reactions) were observed using aqueous solutions of $10 \% \mathrm{KOH}, 28-30 \% \mathrm{NH}_{4} \mathrm{OH}$, and Melzer reagent. Microscopic structures were observed from dried specimens and rehydrated in $5 \%$ potassium hydroxide or $1 \%$ ammoniacal Congo red. For dimensions of microscopic features, a minimum of 50 basidiospores (20 for other structures) were randomly measured at 1,000 $\times$ using a calibrated ocular micrometer on an Olympus CX31 compound microscope. The notations " $[\mathrm{n} / \mathrm{m} / \mathrm{p}]$ " show the number of basidiospores " $n$ " measured from the number of basidiomata " $m$ " of the number of collections " $p$." Dimensions of microscopic structure are presented in the following $(a-)$ $b-c-d(-e)$, in which " $c$ " contains an average, " $b$ " is the $5^{\text {th }}$ percentile, " $d$ " is the $95^{\text {th }}$ percentile, and extreme values " $a$ " and " $e$ " are shown in parentheses. $Q$ is the length/width ratio. Sections of the pileipellis were cut radially, perpendicularly to the surface halfway between the disc and margin of pileus. Sections of stipitipellis or the squamules on the stipe were taken halfway along the stipe length (Li et al., 2011; Hosen et al., 2013; Li et al., 2014; Zhu et al., 2015). All microscopic features were drawn free hand using an Olympus Camera Lucida model U-DA.

\section{DNA Extraction, Polymerase Chain Reaction Amplification, and DNA Sequencing}

Genomic DNA was extracted from fresh tissue preserved in CTAB or approximately $10-15 \mathrm{mg}$ of dried tissue using a $\mathrm{CTAB}$ isolation procedure adapted from Doyle and Doyle (1990). Parts of three protein-coding genes, atp6, tef 1 , and $r p b 2$, were amplified by polymerase chain reaction (PCR) and sequenced. ITS-5.8 region of the nuclear ribosomal DNA was not sequenced because in Boletaceae, ITS commonly shows high levels of intraindividual polymorphisms for indels, thus mostly preventing straightforward phylogenetic analysis and moreover often making cloning necessary to obtain a sequence. The selected protein-coding genes have shown multiple times their usefulness for investigating phylogenetic relationships, at both infrageneric and suprageneric levels (e.g., Wu et al., 2016; Vadthanarat et al., 2019). For the amplification of atp6, ATP6$1 \mathrm{M} 40 \mathrm{~F}$ and ATP6-2M primers were used, with following the protocol and PCR program in Raspé et al. (2016). The primers EF1-983F and EF1-2218R (Rehner and Buckley, 2005) were used to amplify tef 1 gene, and bRPB2-6F and bRPB2-7.1R primers (Matheny, 2005) were used to amplify rpb2 gene. PCR 
products were purified by adding $1 \mathrm{U}$ of exonuclease I and 0.5 U FastAP alkaline phosphatase (Thermo Scientific, St. LeonRot, Germany) and incubating at $37^{\circ} \mathrm{C}$ for $1 \mathrm{~h}$, followed by inactivation at $80^{\circ} \mathrm{C}$ for $15 \mathrm{~min}$. Sequencing was performed by Macrogen Inc. (Korea and the Netherlands) with PCR primers, except for atp6, for which universal primers M13F-pUC(-40) and $\mathrm{M} 13 \mathrm{~F}(-20)$ were used; for tef 1 , additional sequencing was performed with the two internal primers, EF1-1577F and EF11567R (Rehner and Buckley, 2005).

\section{Alignment and Phylogeny Inference}

The sequences were assembled in GENEIOUS Pro v. 6.0.6 (Biomatters). All nucleotide sequences, including nucleotide sequences obtained from GenBank, were aligned using MAFFT version 7 (Katoh and Standley, 2013) on the server accessed at http://mafft.cbrE.jp/alignment/server/.

Before combining the data partitions, topological incongruence between the datasets was assessed using maximum likelihood (ML) and Bayesian inference (BI) separately on each of the four character sets, atp6, tef 1 exons, $r p b 2$ exons, and the three introns of tef $1+$ the intron of $r p b 2$. Paired trees were examined for conflicts involving only nodes with ML bootstrap (BS) $>75 \%$ and Bayesian posterior probabilities (PPs) > 0.95 (Mason-Gamer and Kellogg, 1996; Lutzoni et al., 2004; Reeb et al., 2004). A conflict was assumed to be significant if two different relationships for the same set of taxa (one being monophyletic and the other non-monophyletic) were observed in rival trees. For ML, phylogenetic inference was performed using RAxML (Stamatakis, 2006) on the CIPRES web portal (RAxML-HPC2 on XSEDE; Miller et al., 2009), using the GTRCAT model with 25 categories. Three Butyriboletus species were used as outgroup based on the phylogeny in Vadthanarat et al. (2019). Statistical support of clades was obtained with 1,000 rapid BS replicates. For BI, the best-fit model of substitution among those implementable in MrBayes was estimated separately for each character set using jModeltest (Darriba et al., 2012) on the CIPRES portal, based on the Bayesian information criterion. The selected models were HKY + I + G for atp6, SYM $+\mathrm{I}+\mathrm{G}$ for tef 1 exons, and $\mathrm{K} 80+\mathrm{I}+\mathrm{G}$ for $r p b 2$ exons and intron partition. The Bayesian analysis was performed with MrBayes 3.2.6 software for Windows (Ronquist et al., 2012).

Then, phylogenetic inference of a combined, partitioned dataset was performed, using ML under the same model mentioned above. The phylogenetic tree was inferred from a single analysis with four character sets: atp6, tef 1 exons, $r p b 2$ exons and the three introns of tef $1+$ the intron of $r p b 2$. The same combined dataset was also analyzed by BI, based on the best-fit model mentioned previously. The two runs of five chains were run for 1,000,000 generations and sampled every 200 generations. At the end of the run, the average deviation of split frequencies was 0.008306 , and the potential scale reduction factor values of all parameters were close to 1 . The burn-in phase $(25 \%)$ was estimated by checking the stationarity in the plot generated by the sump command. A total of 7,396 stationary trees were used to reconstruct a $50 \%$ majority rule consensus tree and calculate the Bayesian PPs.

\section{RESULTS}

\section{Phylogenetic Analyses}

Fifty-four sequences from Sutorius collections corresponding to 11 phylogenetic species were newly generated and deposited in GenBank. The combined alignment of the three loci studied contained 190 sequences (32 for atp6, 80 for tef 1,78 for $r p b 2$ ) from 80 specimens (Table 1) and was 2,681 characters long (gaps included) (TreeBase no. 27286).

The single-gene trees were very similar in topology (Supplementary Figures 1-4), except for one supported conflict. In the tef 1 exons tree (Supplementary Figure 2), Sutorius pachypus formed a clade sister to S. australiensis with high support $(\mathrm{BS}=75 \%, \mathrm{PP}=0.92$ ). This is in conflict with the topology of the tree based on the introns character set (Supplementary Figure 4), in which S. pachypus formed a clade sister to Sutorius rubinus (BS $=87 \%, \mathrm{PP}=0.97$ ). Partitioned analyses of a dataset combining atp6, tef 1 exons, and $r p b 2$ exons sequences were also performed (Supplementary Figure 5). Its tree topology showed no conflict to the single partitions of atp6, tef 1 exons, and rpb2 exons trees. Consequently, the combination of four-partition analyses was used to infer the relationship between species in this study.

Maximum likelihood and BI trees of the combined threegene dataset showed similar topologies without any supported conflict (Bootstrap support values, BS $\geq 70 \%$ and $\mathrm{PP} \geq 0.90$; Figure 1). The phylogram of RAxML bipartitions (Figure 1) indicated that all selected Boletaceae formed eight highly supported generic clades $(\mathrm{BS}=100 \%$ and $\mathrm{PP}=1$ for Sutorius, Pulveroboletus, Suillellus, Rubroboletus, Crocinoboletus, and Butyriboletus; BS = 94\% and PP = 1 for the Neoboletus clade). All Sutorius exemplars clustered together in a strongly supported clade, sister to Neoboletus (BS $=70 \%$ and $\mathrm{PP}=0.90$ ). In the Sutorius clade, 17 terminal, species-level clades were resolved with high support, including the three previously described species clades (clade $5=S$. eximius, clade $6=S$. subrufus, and clade $11=S$. australiensis); 7 clades of new, undescribed species originating from Thailand (clades $4,7,8,9,10,12$, and 17), with one clade (clade 13) containing T. maculatoides (WAT25793); and two Thai collections (OR0626 and OR0758). Also, six clades of unnamed species were inferred (clades 1 and 2 from China; clade 3 from Costa Rica; clades 14, 15, and 16 from Togo, Zimbabwe, and Burundi, respectively).

\section{Taxonomy}

Sutorius maculatoides (E. Horak) Vadthanarat, Raspé and Lumyong comb. nov.

MycoBank: MB838055

Figures 2, 3

Basionym: B. maculatus Corner, Boletus in Malaysia: 160, 1972, non-Raddi, Mem. Mat. Fis. Soc. Ital. Sci. Modena, Pt. Mem. Fis. 13: 352. 1807.

Synonymy: T. maculatoides E. Horak, Malayan Forest Records 51: 98, 2011.

Description (based on Thai specimens): Basidiomata smallto medium-sized. Pileus (1.8) 2.4 to $4.6 \mathrm{~cm}$ in diameter, at 
TABLE 1 | List of collections used for DNA analyses of the genus Sutorius, with origin, GenBank accession numbers, and reference(s).

\begin{tabular}{|c|c|c|c|c|c|c|}
\hline Species & Voucher & Origin & atp6 & tef 1 & $r p b 2$ & Reference(s) \\
\hline Butyriboletus appendiculatus & VDKO0193b & Belgium & MG212537 & MG212582 & MG212624 & Vadthanarat et al. (2018) \\
\hline Butyriboletus cf. roseoflavus & OR0230 & China & KT823974 & KT824040 & KT824007 & Raspé et al. (2016) \\
\hline Butyriboletus pseudoregius & VDKO0925 & Belgium & MG212538 & MG212583 & MG212625 & Vadthanarat et al. (2018) \\
\hline Caloboletus firmus & BOS-372 & Belize & - & MK721080 & MK766288 & Kuo and Ortiz-Santana (2020) \\
\hline Caloboletus inedulis & MICH:KUO-07031403 & United States & - & MK721081 & MK766289 & Kuo and Ortiz-Santana (2020) \\
\hline Caloboletus radicans & VDKO1187 & Belgium & MG212540 & MG212584 & MG212626 & Vadthanarat et al. (2018) \\
\hline Caloboletus yunnanensis & HKAS69214 & China & - & KJ184568 & KT990396 & Zhao et al. (2014); Wu et al. (2016) \\
\hline Crocinoboletus cf. laetissimus & OR0576 & Thailand & KT823975 & KT824041 & KT824008 & Raspé et al. (2016) \\
\hline Crocinoboletus rufoaureus & HKAS53424 & China & - & KF112206 & KF112710 & Wu et al. (2014) \\
\hline Neoboletus brunneissimus & HKAS52660 & China & - & KF112143 & KF112650 & Wu et al. (2014) \\
\hline Neoboletus ferrugineus & HKAS77617 & China & - & KT990788 & KT990430 & Wu et al. (2016) \\
\hline Neoboletus ferrugineus & HKAS77718 & China & - & KT990789 & KT990431 & Wu et al. (2016) \\
\hline Neoboletus flavidus & HKAS58724 & China & - & KU974137 & KU974145 & Wu et al. (2016) \\
\hline Neoboletus flavidus & HKAS59443 & China & - & KU974136 & KU974144 & Wu et al. (2016) \\
\hline Neoboletus hainanensis & HKAS63515 & China & - & KT990808 & KT990449 & Wu et al. (2016) \\
\hline Neoboletus hainanensis & HKAS74880 & China & - & KT990790 & KT990432 & Wu et al. (2016) \\
\hline Neoboletus hainanensis & HKAS90209 & China & - & KT990809 & KT990450 & Wu et al. (2016) \\
\hline Neoboletus hainanensis & HKAS59469 & China & - & KF112175 & KF112669 & Wu et al. (2016) \\
\hline Neoboletus junquilleus & AF2922 & France & MG212552 & MG212596 & MG212638 & Vadthanarat et al. (2018) \\
\hline Neoboletus magnificus & HKAS54096 & China & - & KF112149 & KF112654 & Wu et al. (2014) \\
\hline Neoboletus magnificus & HKAS74939 & China & - & KF112148 & KF112653 & Wu et al. (2014) \\
\hline Neoboletus obscureumbrinus & HKAS63498 & China & - & KT990791 & KT990433 & Wu et al. (2016) \\
\hline Neoboletus obscureumbrinus & HKAS77774 & China & - & KT990792 & KT990434 & Wu et al. (2016) \\
\hline Neoboletus obscureumbrinus & HKAS89027 & China & - & KT990794 & KT990436 & Wu et al. (2016) \\
\hline Neoboletus rubriporus & HKAS57512 & China & - & KF112151 & KF112656 & Wu et al. (2014) \\
\hline Neoboletus rubriporus & HKAS83026 & China & - & KT990795 & KT990437 & Wu et al. (2016) \\
\hline Neoboletus rubriporus & HKAS89174 & China & - & KT990796 & KT990438 & Wu et al. (2016) \\
\hline Neoboletus sanguineoides & HKAS57766 & China & - & KT990799 & KT990440 & Wu et al. (2016) \\
\hline Neoboletus sanguineoides & HKAS74733 & China & - & KT990800 & KT990441 & Wu et al. (2016) \\
\hline Neoboletus sanguineoides & HKAS55440 & China & - & KF112145 & KF112652 & Wu et al. (2014) \\
\hline Neoboletus sanguineus & HKAS80823 & China & - & KT990802 & KT990442 & Wu et al. (2016) \\
\hline Neoboletus sanguineus & HKAS68587 & China & - & KF112150 & KF112657 & Wu et al. (2014) \\
\hline Neoboletus sanguineus & HKAS90211 & China & - & KT990804 & KT990444 & Wu et al. (2016) \\
\hline Neoboletus sp. & HKAS76660 & China & - & KF112180 & KF112731 & Wu et al. (2014) \\
\hline Neoboletus sp. & OR0128 & Thailand & MH614686 & MH614734 & MH614781 & Vadthanarat et al. (2019) \\
\hline Neoboletus sp. & HKAS76851 & China & - & KF112144 & KF112651 & Wu et al. (2014) \\
\hline Neoboletus tomentulosus & HKAS77656 & China & - & KT990806 & KT990446 & Wu et al. (2016) \\
\hline Neoboletus tomentulosus & HKAS53369 & China & - & KF112154 & KF112659 & Wu et al. (2014) \\
\hline Neoboletus tomentulosus & HKAS77614 & China & - & KT990805 & KT990445 & Wu et al. (2016) \\
\hline Neoboletus venenatus & HKAS63535 & China & - & KT990807 & KT990448 & Wu et al. (2016) \\
\hline Pulveroboletus aff. ravenelii & ADK4360 & Togo & KT823957 & KT824023 & KT823990 & Raspé et al. (2016) \\
\hline Pulveroboletus aff. ravenelii & ADK4650 & Togo & KT823959 & KT824025 & KT823992 & Raspé et al. (2016) \\
\hline Pulveroboletus aff. ravenelii & HKAS53351 & China & - & KF112261 & KF112712 & Wu et al. (2014) \\
\hline Pulveroboletus fragrans & OR0673 & Thailand & KT823977 & KT824043 & KT824010 & Raspé et al. (2016) \\
\hline Pulveroboletus ravenelii & $\mathrm{REH} 2565$ & United States & KU665635 & KU665636 & KU665637 & Raspé et al. (2016) \\
\hline Pulveroboletus sp. & HKAS57665 & China & - & KF112264 & KF112715 & Wu et al. (2014) \\
\hline Pulveroboletus sp. & HKAS74933 & China & - & KF112262 & KF112713 & Wu et al. (2014) \\
\hline Rubroboletus legaliae & VDKO0936 & Belgium & KT823985 & KT824051 & KT824018 & Raspé et al. (2016) \\
\hline Rubroboletus rhodoxanthus & HKAS84879 & China & - & KT990831 & KT990468 & Wu et al. (2016) \\
\hline Rubroboletus satanas & VDKO0968 & Belgium & KT823986 & KT824052 & KT824019 & Raspé et al. (2016) \\
\hline Suillellus luridus & VDKO0241b & Belgium & KT823981 & KT824047 & KT824014 & Raspé et al. (2016) \\
\hline Suillellus subamygdalinus & HKAS53641 & China & - & KT990841 & KT990478 & Wu et al. (2016) \\
\hline Sutorius aff. eximius & HKAS50420 & China & - & KT990750 & KT990387 & Wu et al. (2016) \\
\hline
\end{tabular}


TABLE 1 | Continued

\begin{tabular}{|c|c|c|c|c|c|c|}
\hline Species & Voucher & Origin & atp6 & tef1 & $r p b 2$ & Reference(s) \\
\hline Sutorius aff. eximius & HKAS52672 & China & - & KF112207 & KF112802 & Wu et al. (2014) \\
\hline Sutorius aff. eximius & HKAS56291 & China & - & KF112208 & KF112803 & Wu et al. (2014) \\
\hline Sutorius aff. eximius & HKAS59657 & China & - & KT990887 & KT990505 & Wu et al. (2016) \\
\hline Sutorius aff. eximius & TWO-986 & Costa Rica & - & MK721180 & MK766383 & Kuo and Ortiz-Santana (2020) \\
\hline Sutorius australiensis & REH9441 & Australia & MG212567 & JQ327032* & MG212652 & Vadthanarat et al. (2018); *Halling et al. (2012) \\
\hline Sutorius australiensis & REH9485 & Australia & - & MK721165 & MK766367 & Kuo and Ortiz-Santana (2020) \\
\hline Sutorius australiensis & REH10021 & Australia & - & MK721166 & MK766368 & Kuo and Ortiz-Santana (2020) \\
\hline Sutorius eximius & REH9400 & United States & MG212568 & JQ327029* & MG212653 & Vadthanarat et al. (2018); *Halling et al. (2012) \\
\hline Sutorius eximius & REH10038 & United States & - & MK721167 & MK766369 & Kuo and Ortiz-Santana (2020) \\
\hline Sutorius maculatoides & OR0758 & Thailand & MN067462 & MN067481 & MN067498 & This study \\
\hline Sutorius maculatoides & WAT25793 & Malaysia & MN067463 & MN067482 & - & This study \\
\hline Sutorius maculatoides & OR0626 & Thailand & MN067461 & MN067480 & - & This study \\
\hline Sutorius mucosus & OR0851 (type) & Thailand & MN067464 & MN067483 & MN067499 & This study \\
\hline Sutorius pachypus & OR0411 (type) & Thailand & MN067465 & MN067484 & MN067500 & This study \\
\hline Sutorius pachypus & SV0098 & Thailand & MN067466 & MN067485 & MN067501 & This study \\
\hline Sutorius pachypus & TW01171 & Thailand & MW194871 & - & - & This study \\
\hline Sutorius pseudotylopilus & OR0378B (type) & Thailand & MH614692 & $\mathrm{MH} 614740$ & MH614787 & Vadthanarat et al. (2019) \\
\hline Sutorius pseudotylopilus & SV0401 & Thailand & MN067467 & MN067486 & MN067502 & This study \\
\hline Sutorius pseudotylopilus & SV0415 & Thailand & MN067468 & MN067487 & MN067503 & This study \\
\hline Sutorius rubinus & OR0379 & Thailand & MH614693 & $\mathrm{MH} 614741$ & MH614788 & Vadthanarat et al. (2019) \\
\hline Sutorius rubinus & OR0403 (type) & Thailand & MN067469 & MN067488 & MN067504 & This study \\
\hline Sutorius rubinus & OR0409 & Thailand & MN067470 & MN067489 & MN067505 & This study \\
\hline Sutorius rubinus & OR1255 & Thailand & MN067471 & MN067490 & MN067506 & This study \\
\hline Sutorius sp. & ADK2396 & Zimbabwe & MN067477 & MN067495 & MN067511 & This study \\
\hline Sutorius sp. & ADK4369 & Togo & MN067478 & MN067496 & MN067512 & This study \\
\hline Sutorius sp. & JD669 & Burundi & MN067479 & MN067497 & MN067513 & This study \\
\hline Sutorius subrufus & FHMU2101 & China & - & MH879730 & MH879747 & Chai et al., 2019 \\
\hline Sutorius subrufus & FHMU2006 & China & - & MH879729 & MH879746 & Chai et al., 2019 \\
\hline Sutorius ubonensis & SV0032 (type) & Thailand & MN067472 & MN067491 & MN067507 & This study \\
\hline Sutorius ubonensis & SV0203 & Thailand & MN067473 & MN067492 & MN067508 & This study \\
\hline Sutorius ubonensis & SV0353 & Thailand & MN067474 & MN067493 & MN067509 & This study \\
\hline Sutorius obscuripellis & OR0949 (type) & Thailand & MN067475 & MN067494 & MN067510 & This study \\
\hline Sutorius vellingae & ECV3603 (type) & Thailand & MN067476 & JQ327033 & - & This study; Halling et al. (2012) \\
\hline
\end{tabular}

Newly generated sequences are shown in bold font.

first hemispherical with straight margin becoming convex with deflexed margin; surface subrugulose, dull, minutely tomentose, at first dark lilac (10-11F3-6) becoming purplish to reddish brown to brown $(8 \mathrm{~F} 6,7 \mathrm{E} 5)$ in age, gradually paler to the margin. Pileus context $4-5 \mathrm{~mm}$ thick halfway to the margin, firm, yellowish off-white (4A2), near the center grayish to purplish white (14-15C-E2) at first, then yellowish white (4A2) in age, with scattered small violet-brown (11F4-6) encrustations. Stipe central, terete to slightly compressed, cylindrical, slightly wider at the base, $2.4-4.5 \times 0.6-1.2 \mathrm{~cm}$; surface finely scabrous with groups of brown to dark brown (7-8F4-6) granulose squamules on purplish gray $(15 \mathrm{C} 2,15 \mathrm{D} 3)$ background; basal tomentum little developed, white. Stipe context solid, pale grayish orange (5B2), virgated with grayish purple (15$16 \mathrm{C} 3)$, with scattered small violet-brown (11F4-6) encrustations. Hymenophore tubulose, subventricose to adnexed to broadly adnexed, depressed around stipe; tubes easily separable, at first grayish brown (7D3) then brownish orange (6C6) with age, 8$9 \mathrm{~mm}$ long halfway to the margin; pores $0.2-0.4(0.7) \mathrm{mm}$ wide at midradius, regular roundish; at first lilac-gray (15-16E3 to $15 \mathrm{C} 2)$ slightly paler near the margin, becoming pale reddish brown (9D-E4) with age. Odor fungoid. Taste mild. Spore print reddish brown (8E/F5).

Macrochemical reactions: $\mathrm{KOH}$ : pale greenish yellow on pileus, pileus context, stipe and stipe context; pale brownish orange on hymenophore. $\mathrm{NH}_{4} \mathrm{OH}$ : pale greenish yellow on pileus, on pileus context bluish white (21A2) when young or negative in mature basidiomes; greenish yellow on stipe; greenish yellow then purplish on stipe context.

Basidiospores [165/3/3] (7.8-) 8.3-10.9-13.2 (-14.1) $\times(3-)$ 3.3-4-4.7 (-5.6) $\mu \mathrm{m}, Q=(2.02-)$ 2.24-2.69-3.18 (-3.41), narrowly ellipsoid to subcylindrical with slight suprahilar depression, thin-walled, smooth, brownish to yellowish hyaline in water, yellowish hyaline in $\mathrm{KOH}$ or $\mathrm{NH}_{4} \mathrm{OH}$, inamyloid. Basidia 4-spored (23-) 23-26-31 (-32) × (10-) 10-11-13 (14) $\mu \mathrm{m}$, with sterigmata up to $4 \mu \mathrm{m}$ long, clavate, hyaline, inamyloid. Cheilocystidia (19-) 19-22-27 (-27) × (6-) 6-8-9 (-9) $\mu \mathrm{m}$, frequent, fusiform, thin-walled, hyaline. Pleurocystidia 


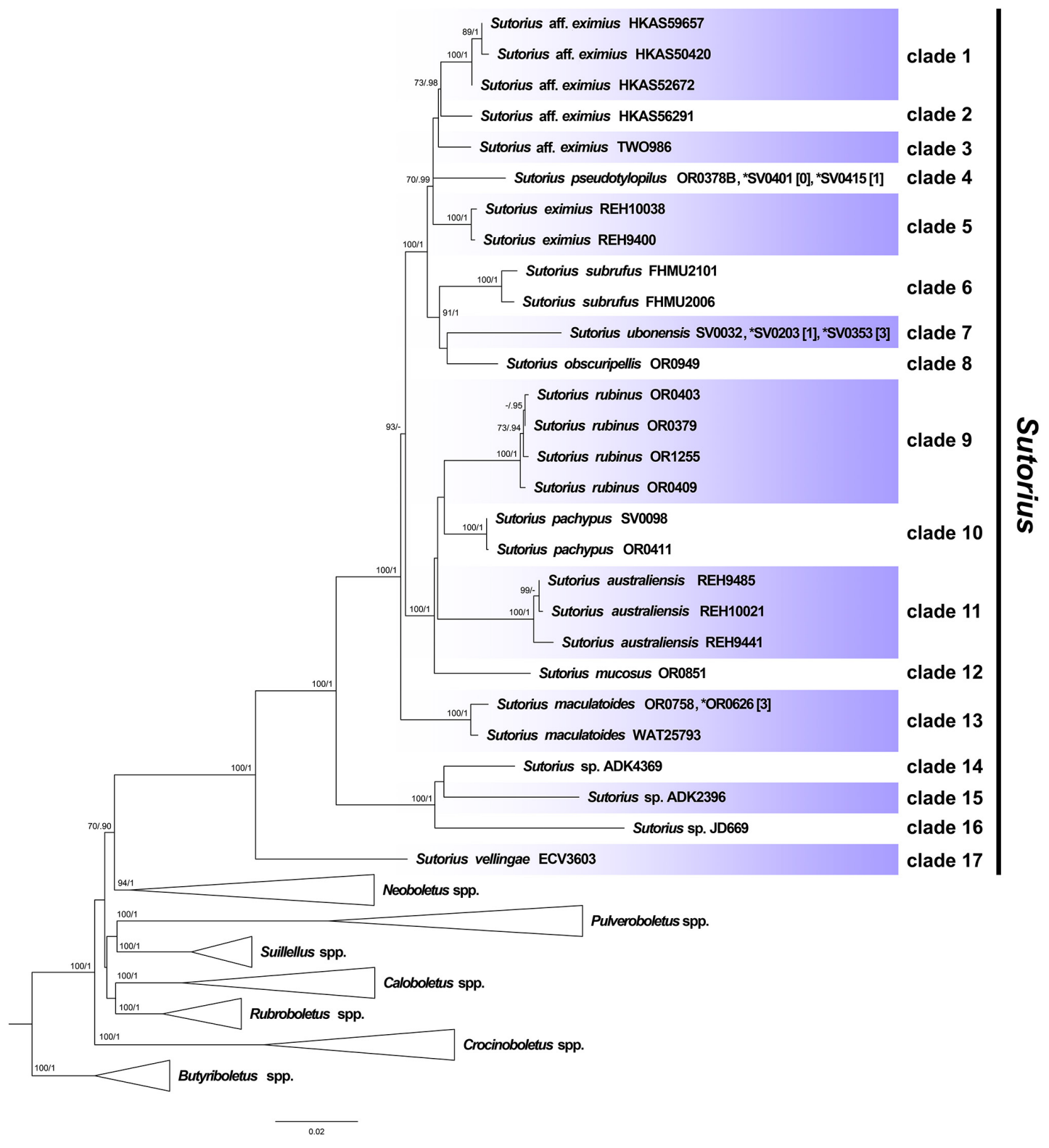

FIGURE 1 | Phylogenetic tree inferred from the three-gene dataset (atp6, rpb2 and tef1), of Sutorius species and selected Boletaceae in Pulveroboletus group, using Maximum Likelihood and Bayesian Inference methods (ML bipartition tree is presented). The three Butyriboletus species were used as outgroup. All generic clades, excluding Sutorius, that were highly supported were collapsed. Seventeen species-level clades within Sutorius are indicated with label. Bootstrap support values (BS $\geq 70 \%)$ and posterior probabilities ( $\mathrm{PP} \geq 0.90)$ are shown above the supported branches. The star (*) indicates additional collections with exactly identical sequences or sequences differing only by heteromorphisms in tef 1 (with the number of heteromorphisms mentioned in square brackets [ ]).

(32-) 32-38-45 (-45) × (6-) 6-8-10 (-10) $\mu \mathrm{m}$, infrequent, narrowly fusiform, thin-walled, hyaline. Hymenophoral trama divergent becoming boletoid in age, 21-52 $\mu \mathrm{m}$ wide, with regular mediostratum 9-22 $\mu \mathrm{m}$ wide. Pileipellis a sub-ixotrichoderm to intricate trichoderm, 135-212 $\mu \mathrm{m}$ thick, terminal cells (12$55 \times 5-11 \mu \mathrm{m})$ fusiform with slightly tapering apex, pale yellowish brown in water, mostly hyaline to pale yellowish brown in $\mathrm{KOH}$ or $\mathrm{NH}_{4} \mathrm{OH}$, with scattered loose crystals. Pileus context made of strongly interwoven, hyaline, thin-walled hyphae, 8$13 \mu \mathrm{m}$ wide, with scattered loose crystals. Stipitipellis a disrupted hymeniderm, 100-120 $\mu \mathrm{m}$ thick, composed of parallel hyphae, with terminal cell $11-33 \times 7-10 \mu \mathrm{m}$ of thin-walled, hyaline hyphae, giving rise to clusters of caulocystidia and basidiole-like cells, with scattered loose crystals. Caulocystidia (18-) 18-26-37 $(-38) \times(8-)$ 8-11-15 (-15) $\mu \mathrm{m}$, frequent, fusiform to broadly fusiform with subacute apex, thin-walled, hyaline. Stipe context composed of parallel, 4- to 12- $\mu \mathrm{m}$-wide hyphae, with scattered loose crystals. Clamp connections not seen in any tissue.

Microscopic description (based on WAT25793): Basidiospores (11.4-) 11.5-12.1-12.7 (-13.5) × (4.1-) 4.2-4.5-4.8 (-4.9) $\mu \mathrm{m}$, 


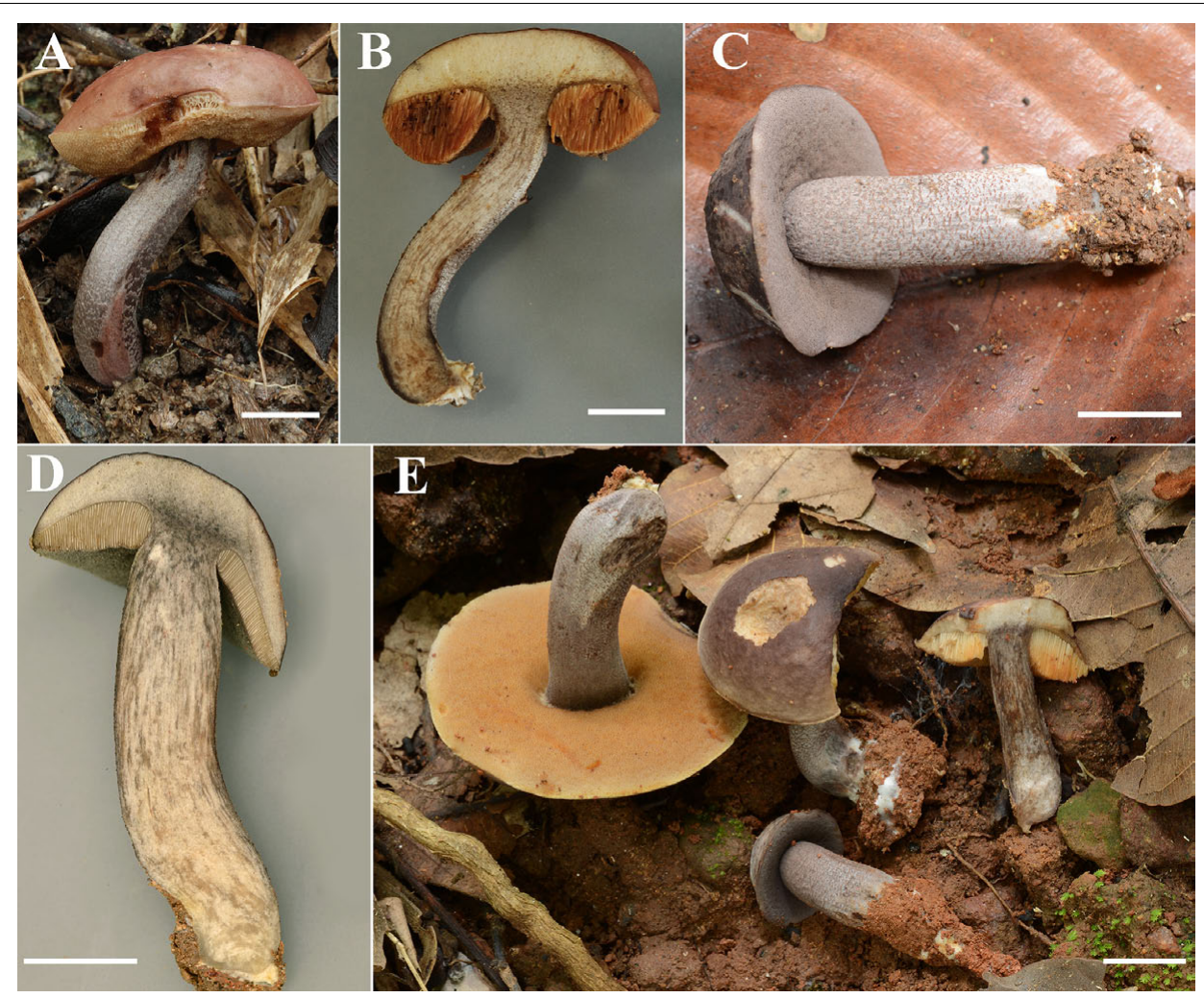

FIGURE 2 | (A-E) Fresh basidiomata of Sutorius maculatoides (A,B: OR0626; C,D: OR0754; E: OR758). Scale bars: (A-E) = 1 cm.

$Q=(2.42-)$ 2.42-2.68-2.94 (-3.05), $N=72$, narrowly ellipsoid to subcylindrical with slight suprahilar depression, thin-walled, smooth, brownish to yellowish hyaline in water, yellowish hyaline in $\mathrm{KOH}$ and $\mathrm{NH}_{4} \mathrm{OH}$, inamyloid. Basidia 4-spored (21-) 2124-28 (-29) × (9-) 10-11-12 (-12) $\mu \mathrm{m}$, with sterigmata up to $3 \mu \mathrm{m}$ long, clavate, yellowish hyaline to yellowishbrown in $\mathrm{KOH}$, inamyloid. Cheilocystidia (15-) 16-21-24 (24) $\times(6-)$ 6-7-8 (-8) $\mu \mathrm{m}$, frequent, fusiform, thin-walled hyaline. Pleurocystidia (31-) 31-36-43 (-43) × (5-) 5-6-7 (-7) $\mu \mathrm{m}$, quite rare, narrowly fusiform, thin-walled, hyaline. Pileipellis a sub-ixotrichoderm to intricate trichoderm, 67- to 132- $\mu \mathrm{m}$-thick, thin-walled hyphae, with fusiform terminal cells (11-53 $\times 6-11 \mu \mathrm{m})$ with slightly tapering apex. Pileus context made of moderately interwoven hyaline hyphae, 6-13 $\mu \mathrm{m}$ wide. Stipitipellis a disrupted hymeniderm, 65-85 $\mu \mathrm{m}$ thick, composed of parallel hyphae, with rounded terminal cell $14-40 \times 5-$ $10 \mu \mathrm{m}$ of thin-walled, hyaline hyphae; at places the terminal cells grouped with caulocystidia into clusters, with scattered loose crystals. Stipe context composed of parallel 4- to 14$\mu \mathrm{m}$-wide hyaline, thin-walled hyphae. Caulocystidia (20-) 2028-37 (-37) × (7-) 8-11-15 (-15) $\mu \mathrm{m}$, frequent, fusiform to broadly fusiform with subacute apex, thin-walled, hyaline. Clamp connections not seen in any tissue.

Habitat: Solitary to gregarious on soil, in hill forest dominated by Dipterocarpus obtusifolius, Dipterocarpus tuberculatus, Shorea obtusa, and Shorea siamensis.

Distribution: Malaysia, Singapore, and Thailand.
Collections examined: MALAYSIA: Forest Research Institute Kepong, MNS foray along rovers' trail, 27 February 1994, Roy Watling, WAT25793 (E). THAILAND: Chiang Mai Province: Doi Suthep-Pui National Park, $18^{\circ} 48^{\prime} 47^{\prime \prime} \mathrm{N}-98^{\circ} 56^{\prime} 11^{\prime \prime} \mathrm{E}$, elev. 540 m, May 18, 2015, Olivier Raspé, OR0626 (BKF, CMUB); -ibid., $18^{\circ} 47^{\prime} 37^{\prime \prime} \mathrm{N}-98^{\circ} 55^{\prime} 41^{\prime \prime}$ E, elev. $770 \mathrm{~m}$, May 24, 2015, Olivier Raspé and Santhiti Vadthanarat, OR0754 (BKF, CMUB); -ibid., $18^{\circ} 47^{\prime} 38^{\prime \prime} \mathrm{N}-98^{\circ} 55^{\prime} 42^{\prime \prime}$ E, elev. $770 \mathrm{~m}$, May 26, 2015, Olivier Raspé and Santhiti Vadthanarat, OR0758 (BKF, CMUB); Mae On District, Ban Huay Kaew community forest, $18^{\circ} 52^{\prime} 7.15^{\prime \prime} \mathrm{N}-99^{\circ} 17^{\prime} 38.92^{\prime \prime}$ E. $750 \mathrm{~m}$, July 17, 2017, Olivier Raspé, OR1414 (CMUB, BR).

Notes: Corner (1972) described B. maculatus from Malaysia. However, it was a later non-homotypic homonym of $B$. maculatus Raddi described in 1807. Consequently, Horak (2011) gave the new epithet maculatoides. He also transferred the species to Tylopilus. Here, we present phylogenetic evidence supporting placement in Sutorius. In our phylogenetic inference (Figure 1), all S. maculatoides collections clustered together in a wellsupported terminal clade (clade 13), within the Sutorius clade. The macromorphological and micromorphological characters of $S$. maculatoides also support its position in Sutorius. Therefore, the new combination $S$. maculatoides is proposed for T. maculatoides. In our phylogeny, among the S. maculatoides specimens, the Thai collections (OR0626 and OR0758) were molecularly slightly divergent from the Malaysian specimen (WAT25793). Being the amount of divergence is lower than 
Vadthanarat et al.

Eight New Taxa of Sutorius

OO

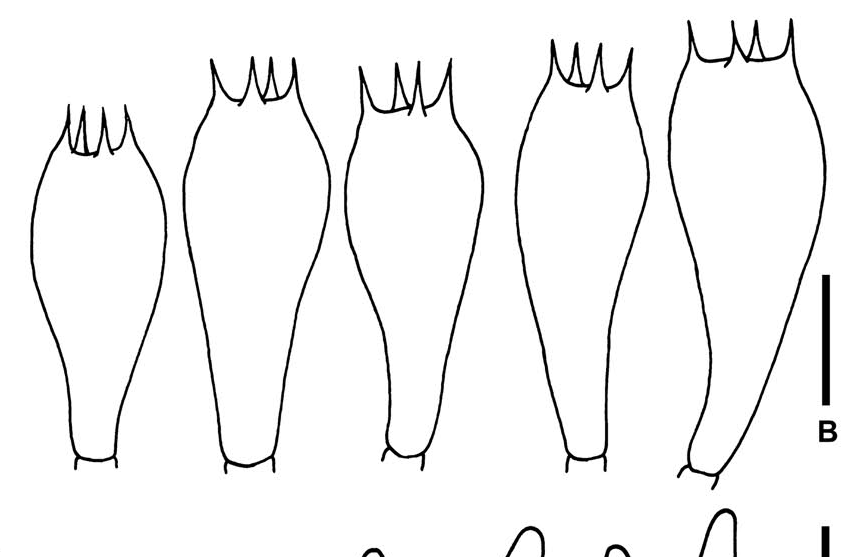

OU

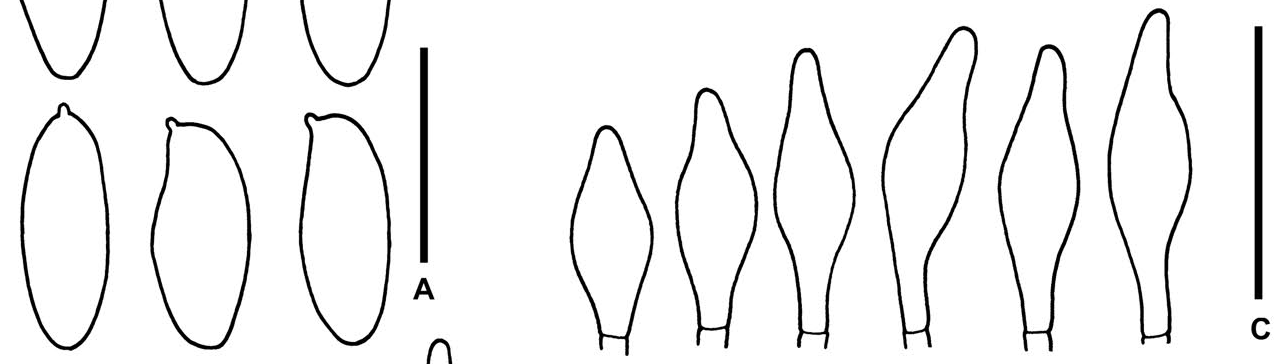
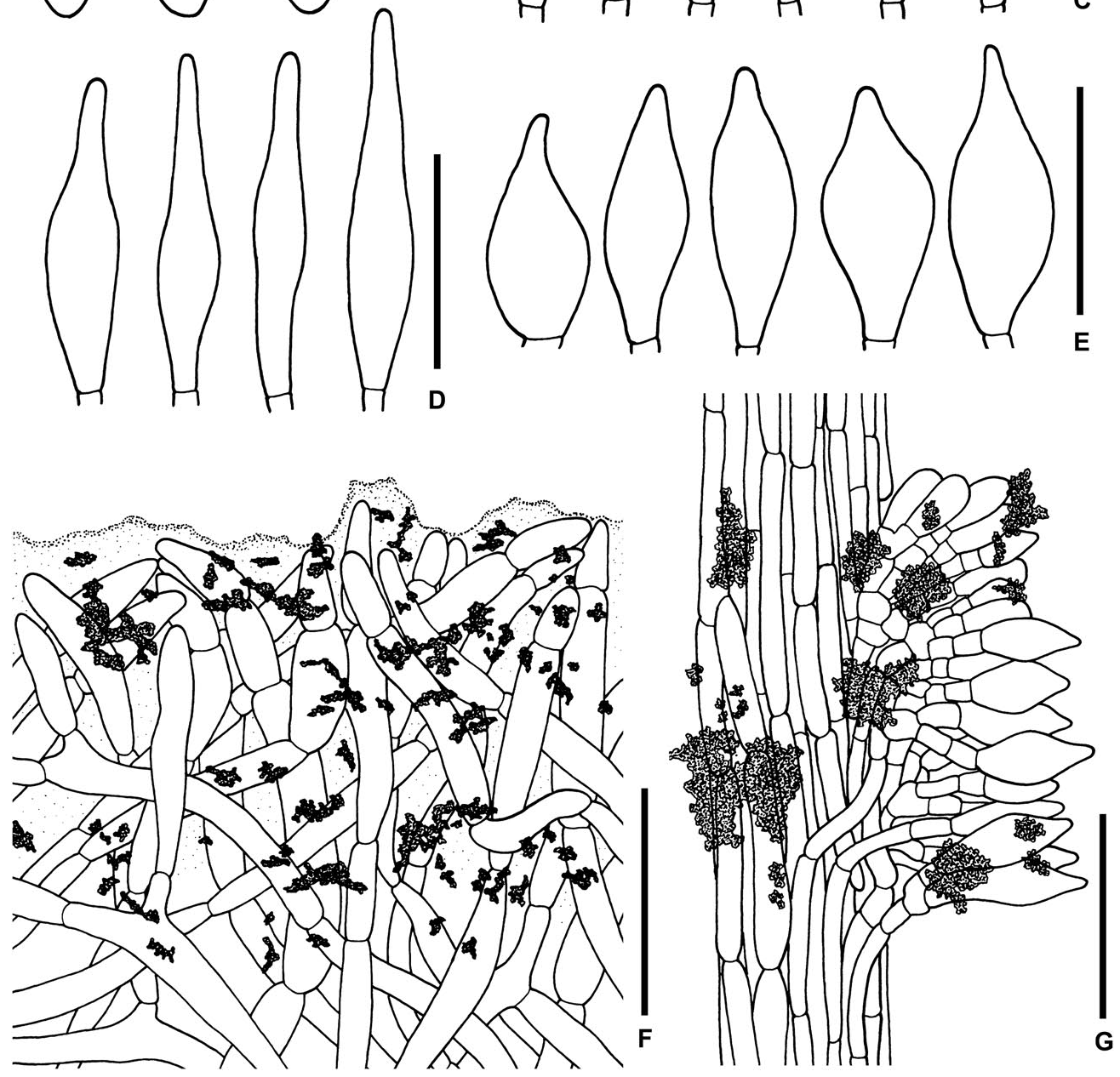

FIGURE 3 | Microscopic features of Sutorius maculatoides. (A) Basidiospores. (B) Basidia. (C) Cheilocystidia. (D) Pleurocystidia. (E) Caulocystidia. (F) Pileipellis.

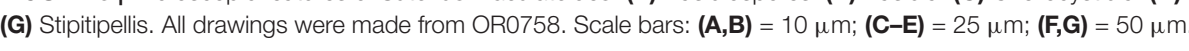

Frontiers in Microbiology | www.frontiersin.org

8

April 2021 | Volume 12 | Article 643505 
the one observed between sister species, we consider those differences in the range of intraspecific genetic variability and compatible with conspecific, geographically distant populations.

Sutorius maculatoides is characterized by the following characteristics: dark lilac pileus with lilac gray pores at first, with age becoming reddish brown and reddish pale brown, respectively; presence of cheilocystidia and infrequent pleurocystidia; pileipellis a sub-ixotrichoderm composed of moderately interwoven hyphae, with fusiform terminal cells that slightly taper at the apex.

In this study, $S$. maculatoides is redescribed based on specimens from Thailand (OR0626 and OR0758) and Malaysia (WAT25793), which were compared to the description of $T$. maculatoides E. Horak (Horak, 2011). Microcharacteristics of Watling's specimen were similar to Horak's description including size and shape of basidiospores, basidia, and pileipellis. However, the cheilocystidia in Watling's specimen $(15-24 \times 6-8 \mu \mathrm{m})$ are shorter than in Horak's description $(35-45 \times 6-11 \mu \mathrm{m})$, but Horak did not separately describe the length of cheilocystidia and pleurocystidia. He also did not mention the frequency of both types of cystidia. By comparison, microscopic characters between Watling's specimen and the Thai specimens were almost identical. However, the minimum value of the length of basidiospores in Watling's specimen $(11.4-13.5 \times 4.1-4.9 \mu \mathrm{m})$ is higher than for the Thai specimens $(7.8-14.1 \times 3-5.6 \mu \mathrm{m})$. The lower minimum value of length of basidiospores in Thai specimens could be explained by small quantitative differences between geographically distant populations or the less mature stage of some basidiomata from Thai collections. Moreover, according to our observations on Sutorius, this genus usually shows high within-species variation in spore size, which was also noted originally by Halling et al. (2012).

Sutorius mucosus Vadthanarat, Raspé and Lumyong sp. nov. MycoBank: MB838057

Figures 4A-C, 5

Typification: THAILAND: Chiang Mai Province: Mae Taeng District, Pha Deng village, $19^{\circ} 6^{\prime} 31^{\prime \prime} \mathrm{N}-98^{\circ} 44^{\prime} 30^{\prime \prime} \mathrm{E}$, elev. $1,050 \mathrm{~m}$, July 29, 2014, Olivier Raspé and Anan Thawthong, OR0851 (MFLU: holotype; BR: isotype).

Etymology: from Latin mucosus, means mucus, referring to a strongly gelatinized pileipellis.

Description: Basidiomata medium-sized. Pileus $9 \mathrm{~cm}$ in diameter, planoconvex with straight margin, margin slightly exceeding (1 mm); surface subrugulose, dull, waxy to subviscid, almost smooth to minutely tomentose under the lens, reddish brown (8E/F8). Pileus context 8-10 $\mathrm{mm}$ halfway to the margin, soft, dull yellowish white (4A2), with scattered small groups of reddish brown (9F5-7) encrustations, slightly reddening (5A2-3) when cut. Stipe central, terete, cylindrical, $\times 1.2 \mathrm{~cm}$; surface subscabrous, with granulose squamules, purplish brown to dark brown (10-11F4-7), uplifted by pale brown (5B/C35) hyphae; stipe context solid, slightly turgid, beige $(4 \mathrm{~B} / \mathrm{C} 3)$. Hymenophore tubulose, ventricose; tubes easily separable, pale orange (5A2-3), 7-9 $\mathrm{mm}$ long halfway to the margin; pores $0.2-0.3(0.5) \mathrm{mm}$ wide at midradius, roundish to angular near the stipe, pore topography irregular, brown to brownish gray
(7B4-5 to 8D/E4). Odor and taste not observed. Spore print reddish brown (8E/F8).

Macrochemical reactions: $\mathrm{KOH}$ : yellowish on pileus context.

Basidiospores (10.4-) 10.8-13.8-15 (-15.4) × (4.3-) 4.6-5$5.5(-5.6) \mu \mathrm{m}, Q=(2.24-)$ 2.47-2.76-3.04 (-3.06), $N=55$, narrowly ellipsoid to subcylindrical with slight suprahilar depression, thin-walled, smooth, brownish to yellowish hyaline in water, yellowish hyaline in $\mathrm{KOH}$ or $\mathrm{NH}_{4} \mathrm{OH}$, inamyloid. Basidia 4-spored (23-) 23-26-30 (-30) × (10-) 10-11-13 $(-13) \mu \mathrm{m}$, with sterigmata up to $4 \mu \mathrm{m}$ long, clavate, hyaline, inamyloid. Cheilocystidia and Pleurocystidia not seen. H. trama slightly divergent to boletoid 17-42 $\mu \mathrm{m}$ wide, with subregular mediostratum 7-12 $\mu \mathrm{m}$ wide. Pileipellis an ixotrichoderm, 75$125 \mu \mathrm{m}$ thick, made of moderately interwoven, gelatinized, hyaline hyphae, with terminal cells 11-37 $\times$ 7-11 $\mu \mathrm{m}$, fusiform to utriform with rounded to tapping apex, pale yellowish to brownish hyaline in water, mostly hyaline to yellowish hyaline in $\mathrm{KOH}$ or $\mathrm{NH}_{4} \mathrm{OH}$, with scattered loose crystals. Pileus context composed of strongly gelatinized hyaline hyphae 8-16 $\mu \mathrm{m}$ wide, at places with scattered loose crystals. Stipitipellis a disrupted hymeniderm 200-450 (600) $\mu \mathrm{m}$ thick composed of parallel hyphae, with terminal cell 15-29 × 6$9 \mu \mathrm{m}$ of thin-walled, hyaline hyphae, giving rise to clusters of basidiole-like cells and caulocystidia, with scattered loose crystals. Caulocystidia (19-) 19-27-36 (-36) × (8-) 8-11-15 (-15) $\mu \mathrm{m}$, not frequent, fusiform to broadly fusiform, thinwalled, hyaline. Stipe context composed of parallel, 8- to 12$\mu \mathrm{m}$-wide gelatinized hyphae, somewhere covered with some encrustations on the wall, with scattered loose crystals. Clamp connections not seen.

Habitat: Solitary on soil in evergreen hill forest dominated by Castanopsis spp., Lithocarpus spp. mixed with D. obtusifolius, Dipterocarpus costatus, Sh. obtusa, and Sh. siamensis.

Distribution: Chiang Mai Province, Northern Thailand.

Notes: S. mucosus description is based on a single collection (OR0851); however, the strongly gelatinized pileipellis is a strikingly unique character, which, together with the phylogenetic evidence, which resolved this specimen as an isolated branch, within the Sutorius lineage, supports our interpretation of OR0851 as a new, distinct species. Other important characters that allow differentiating $S$. mucosus are the fusiform to utriform pileipellis terminal cells with rounded to subacute apex and the lack of cheilocystidia and pleurocystidia.

Phylogenetically, S. mucosus is resolved as an isolated branch, within a clade comprising three other distinct Sutorius species, including S. rubinus (clade 9), S. pachypus (clade 10), and S. australiensis (clade 11). However, they are morphologically different, and none of them has a strong gelatinized pileipellis, which is the most striking diagnostic character of S. mucosus. The other differences are as follows: S. pachypus has, on average, a wider stipe (2.5-4 cm wide) and paler stipe surface, presence of cheilocystidia and pleurocystidia, and palisadodermal pileipellis composed of subcylindrical terminal cells with rounded to subacute apex. S. australiensis has a trichoderm pileipellis with elongated to cylindrical hyphae with obtuse apex terminal cells. S. rubinus also has a trichoderm pileipellis but with different shapes of terminal cells, which are fusiform to broadly 

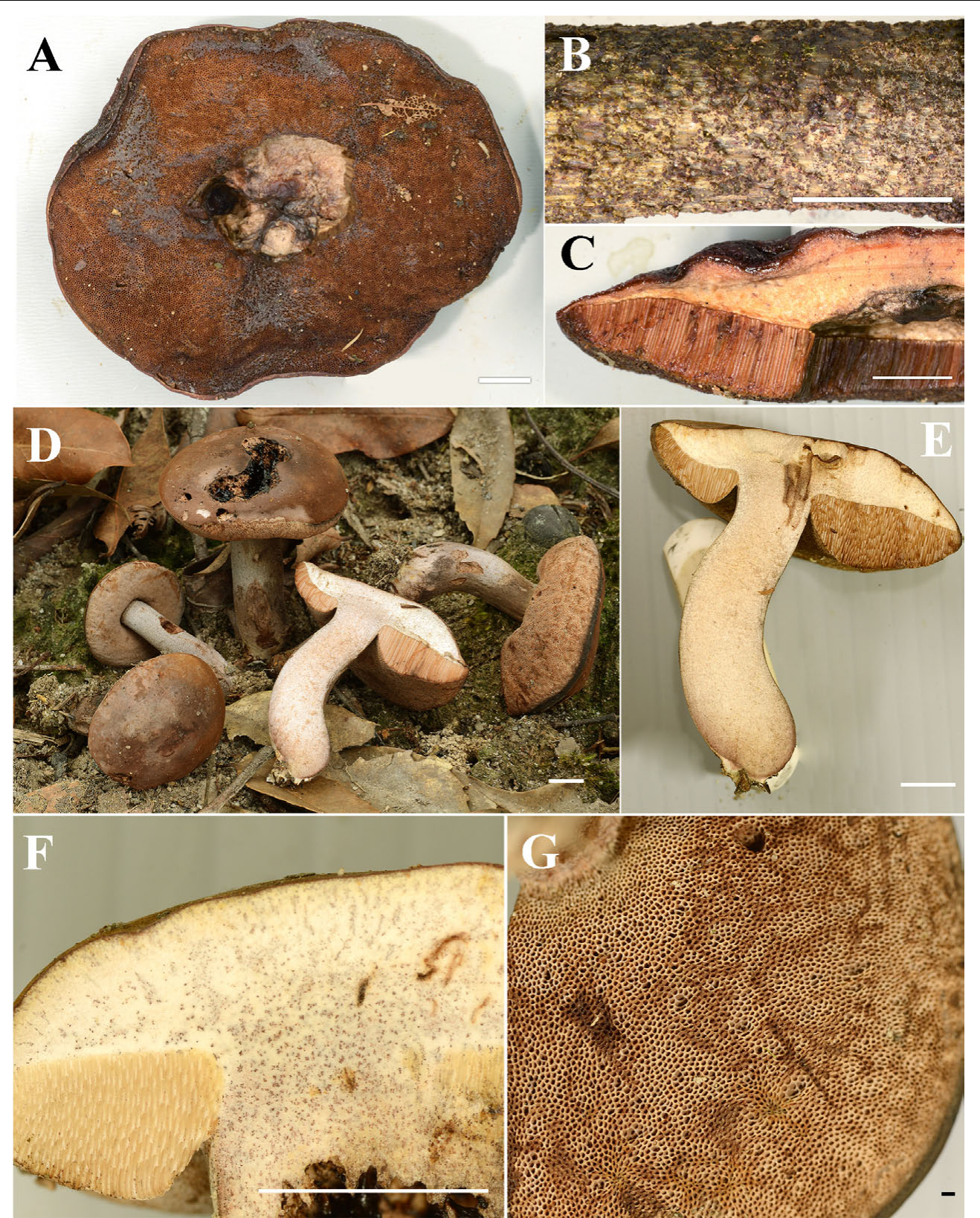

FIGURE 4 | (A-C) Fresh basidiomata of Sutorius mucosus specimen voucher OR0851 holotype (A: pores surface, B: stipe surface, C: slimy pileus and context with tubes). (D-G) Fresh basidiomata of Sutorius obscuripellis OR0949 holotype. Scale bars: $(\mathbf{A}-\mathbf{F})=1 \mathrm{~cm}, \mathbf{( G )}=1 \mathrm{~mm}$.

fusiform with acuminate to subacute or acute apex, and presence of cheilocystidia.

Sutorius obscuripellis Vadthanarat, Raspé and Lumyong sp. nov. MycoBank: MB838059

Figures 4D-G, 6

Typification: THAILAND: Chiang Mai Province: Mae On District, Pok village, $18^{\circ} 53^{\prime} 11^{\prime \prime} \mathrm{N}-99^{\circ} 22^{\prime} 11^{\prime \prime}$ E, elev. $710 \mathrm{~m}$, July 29, 2015, Olivier Raspé, OR0949 (CMUB: holotype, BR: isotype).

Etymology: from Latin obscurus and pellis, referring to the dark pileipellis.
Description: Basidiomata small- to medium-sized. Pileus (3.5) $4.5-6 \mathrm{~cm}$ in diameter convex to planoconvex, with straight margin; surface even, minutely and densely matted tomentose, dull, violet-brown to dark brown (10F5-6 to 9E4), with patchy color variation, sometimes gradually paler to margin. Pileus context 6-9 $\mathrm{mm}$ thick halfway to the margin, yellowish white (4A2), more brownish near the pellis, slowly turning pale red-brown when cut, with scattered small groups of reddish brown to dark brown (9E4-5, 9F5-6) encrustations. Stipe clavate to cylindrical (3.5) $3.8-5.5 \times 0.8-1.7 \mathrm{~cm}$; surface dull, even to finely scabrous, violet-gray $(18 \mathrm{D} 2,16 \mathrm{E} 2)$ with 


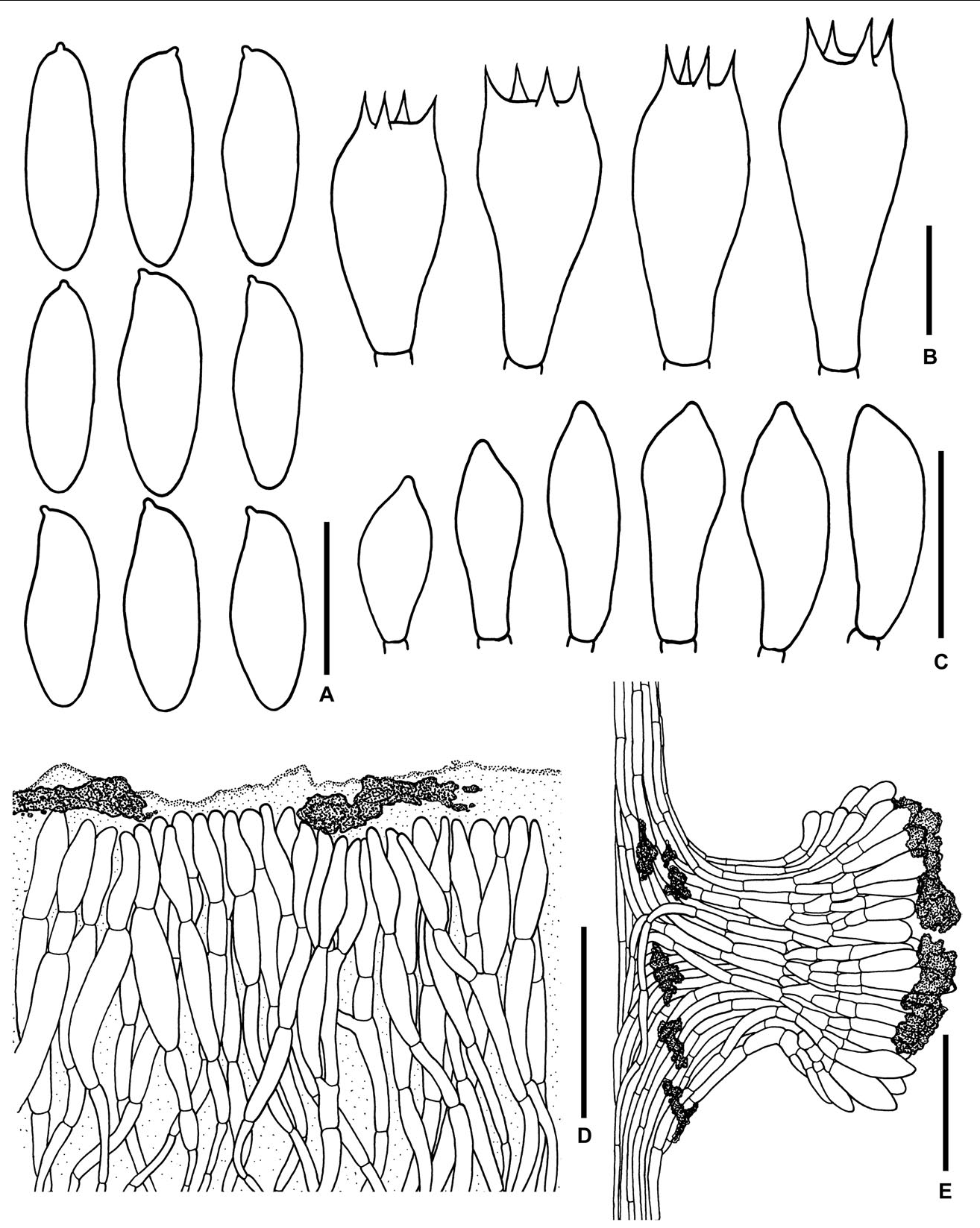

FIGURE 5 | Microscopic features of Sutorius mucosus. (A) Basidiospores, (B) Basidia, (C) Caulocystidia, (D) Pileipellis, (E) Stipitipellis. All drawings were made from OR0581. Scale bars: $(\mathbf{A}, \mathbf{B})=10 \mu \mathrm{m} ;(\mathbf{C})=25 \mu \mathrm{m} ;(\mathbf{D}, \mathbf{E})=50 \mu \mathrm{m}$.

violet-brown (10E43-5) granulose squamules; basal tomentum off-white to yellowish white. Stipe context solid, fleshy fibrous, light purplish brown (12B2), more purplish at the base, with scattered small groups of reddish brown to dark brown (9E45, 9F5-6) encrustations. Hymenophore tubulose, adnexed to adnate, depressed around stipe, ventricose; tubes orange-gray (6B2), 6-13 mm long, easily separable, turning red-brown on bruising; pores $0.2-0.5 \mathrm{~mm}$ wide at midradius, roundish, regularly arranged, with subregular pore surface, pale violetgray (9-10B3), becoming paler with age, slightly reddish brown on bruising, with scattered small groups of reddish brown to dark brown encrustations, composite pores uncommon. Odor not recorded. Taste slightly bitter at first, then mild. Spore print not recorded.

Macrochemical reactions: $\mathrm{KOH}$ : dull yellow on pileus; yellow or pale yellow on pileus context, stipe, stipe context, and hymenophore. $\mathrm{NH}_{4} \mathrm{OH}$ : yellowish with only slight violet aura on pileus; yellowish on stipe and pileus context; negative on hymenophore and stipe context.

Basidiospores (9.6-)9.8-10.8-12.2 (-13.5) × (3.6-) 3.8-4.24.6 (-5.1) $\mu \mathrm{m}, Q=(2.19-)$ 2.34-2.55-2.83 (-2.97), $N=72$, narrowly ellipsoid to subcylindrical with slightly suprahilar 

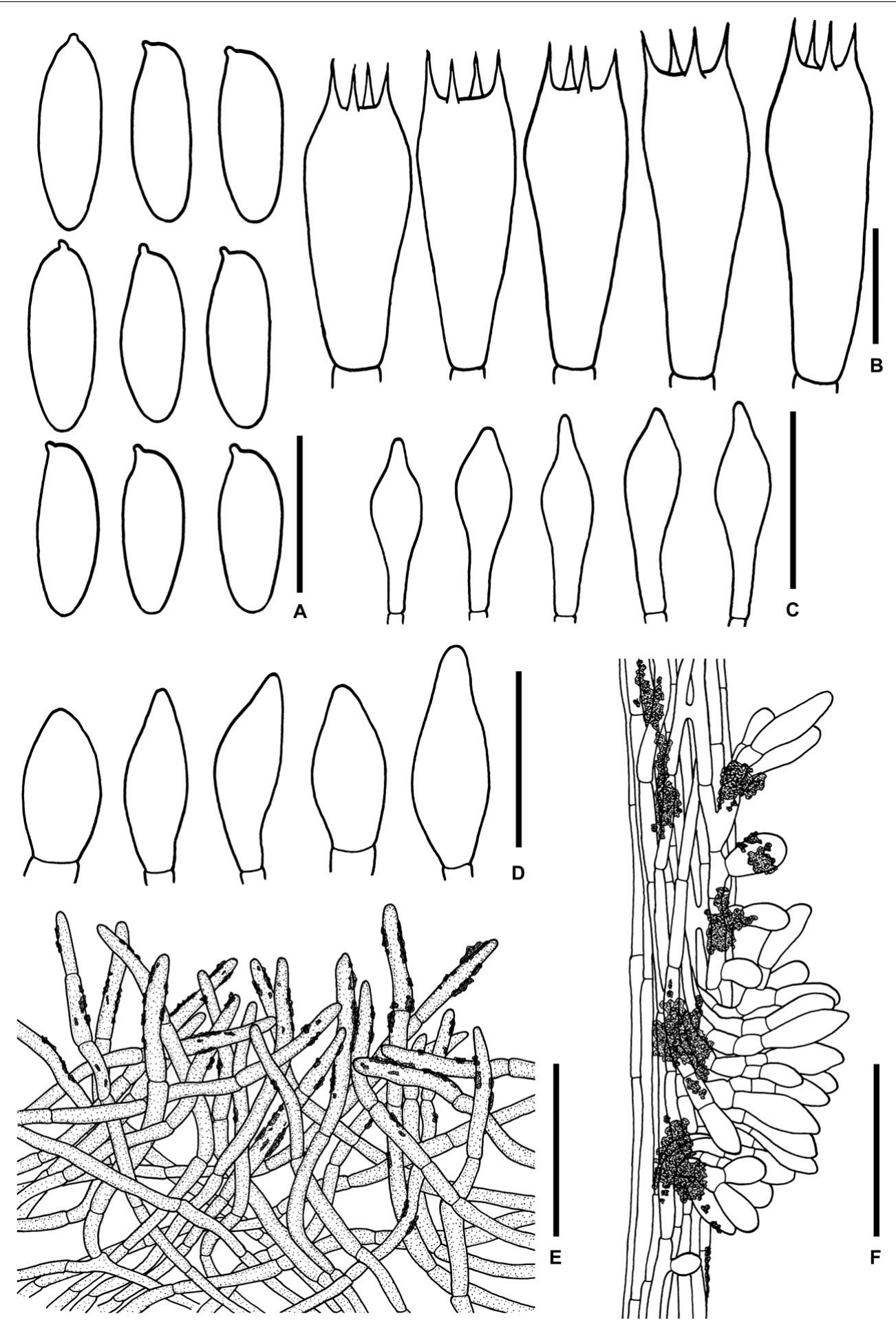

FIGURE 6 | Microscopic features of Sutorius obscuripellis. (A) Basidiospores. (B) Basidia. (C) Cheilocystidia. (D) Caulocystidia. (E) Pileipellis. (F) Stipitipellis. All drawings were made from OR0949. Scale bars: $(\mathbf{A}, \mathbf{B})=10 \mu \mathrm{m} ; \mathbf{( C , D )}=25 \mu \mathrm{m} ; \mathbf{( E , F )}=50 \mu \mathrm{m}$.

depression, thin-walled, smooth, brownish to yellowish hyaline in water, yellowish hyaline in $\mathrm{KOH}$ and $\mathrm{NH}_{4} \mathrm{OH}$, inamyloid. Basidia 4-spored (23-) 23-26-30 (-30) × (8-) 8-10-11 (-11) $\mu \mathrm{m}$, with sterigmata up to $5 \mu \mathrm{m}$ long, clavate, hyaline, inamyloid.
Cheilocystidia (18-) 19-24-30 (-31) × (6-) 6-7-9 (-9) $\mu \mathrm{m}$, frequent, fusiform, thin-walled, hyaline. Pleurocystidia not seen. $H$. trama divergent to boletoid, 47-91 $\mu \mathrm{m}$ wide; with $12-$ $48 \mu \mathrm{m}$ wide of regular mediostratum. Pileipellis an intricate 
trichoderm, 100-170 $\mu \mathrm{m}$ thick, made of loosely interwoven terminal cells $17-65 \times 3-6 \mu \mathrm{m}$, with subacute to rounded apex, brown to dark in water especially the terminal cells, less brown in $\mathrm{KOH}$ or $\mathrm{NH}_{4} \mathrm{OH}$. Pileus context composed of moderately interwoven, 6- to 12 (15)- $\mu \mathrm{m}$-wide hyphae, brownish to yellowish hyaline, with some scattered parietal encrustations and loose crystals. Stipitipellis a disrupted hymeniderm 100$125 \mu \mathrm{m}$ thick, composed of parallel hyphae, anastomosing at places, with terminal cell 10-24 $\times 6-12 \mu \mathrm{m}$ of thin-walled hyaline hyphae, giving rise to clusters of caulocystidia and basidiole-like cells, with scattered loose crystals. Caulocystidia (19-) 20-26-38 (-38) × (6-) 6-11-20 (-22) $\mu \mathrm{m}$, broadly fusiform, thin-walled, hyaline. Stipe context composed of parallel 5- to 8 (12)- $\mu$ m-wide hyphae, with scattered loose crystals. Clamp connections not seen in any tissue.

Habitat: On soil, gregarious to fasciculate in hill forest dominated by Castanopsis spp. Lithocarpus spp. mixed with some Dipterocarpus spp. and regularly subject to fire.

Distribution: Chiang Mai Province, Northern Thailand.

Notes: Based on a single collection (OR0949), S. obscuripellis is characterized by the following combination of characteristics: basidiomata small- to medium-sized; pale violet-gray pores color; lack of pleurocystidia; Pileipellis an intricate trichoderm composed of brown to dark hyphae with cylindrical terminal cells with subacute to rounded apex, showing scattered small parietal brownish yellow to reddish pale to dark brown encrustations. Phylogenetically, S. obscuripellis formed a clade (clade 8) sister to Sutorius ubonensis (clade 7) and S. subrufus (clade 6). However, the latter two species are morphologically different from S. obscuripellis as follows: S. ubonensis has larger basidiomata, darker pores, and basidiomata especially when young, pleurocystidia, and a slightly gelatinized tomentose pileipellis, and has so far only been found in dry dipterocarp forest in Ubon Ratchathani Province, in northeastern Thailand. S. subrufus has larger basidiomata, a stipe surface and context turning more reddish when injured, presence of pleurocystidia, and a trichoderm pileipellis composed of cylindrical hyphae and clavate or subclavate terminal cells with obtuse apex, colorless to yellowish in $\mathrm{KOH}$ (Chai et al., 2019), whereas S. obscuripellis has the pileipellis composed of brown to dark brown hyphae, colorless to brownish yellow to pale brown in $\mathrm{KOH}$.

Sutorius pachypus Vadthanarat, Raspé and Lumyong sp. nov. MycoBank: MB838060

\section{Figures 7A-E, 8}

Typification: THAILAND: Chiang Mai Province: Mae Taeng District, Behind Wat Pah Deng, $19^{\circ} 06^{\prime} 38^{\prime \prime} \mathrm{N}-98^{\circ} 44^{\prime} 32^{\prime \prime} \mathrm{E}$, elev. 1,055 m, June 7, 2012, Olivier Raspé and Komsit Wisitrassameewong, OR0411 (MFLU: holotype; BR: isotype).

Etymology: from Greek means "thick" referring to the wide stipe.

Description: Basidiomata medium-sized. Pileus $4.5-9 \mathrm{~cm}$ in diameter; at first hemispherical then convex to planoconvex with deflexed, slightly exceeding margin (1-2 mm); surface subrugulose, dull, minutely tomentose, at first dark reddish brown to dark brown (9F5-6) becoming pale reddish brown (8-9D/E4-7), gradually paler to the margin. Pileus context
$1.5-1.7 \mathrm{~cm}$ halfway to the margin, pale grayish orange (6A2) to purplish gray $(17 \mathrm{C} 3,17 \mathrm{D} 4)$ at place, with scattered small groups of dark reddish brown (9F5-6) encrustations, unchanging or slightly reddening when cut. Stipe central, wide, cylindrical (5.5) $6-6.5 \times 2.5-4 \mathrm{~cm}$; surface even to finely scabrous, dry, reddish to grayish gray (10B-D2) darker at the base $(10 \mathrm{D} / \mathrm{E} 3)$, with pale violet gray (10D2) to violet brown (10F3-4) granulose squamules; basal tomentum little developed, white. Stipe context solid, orange white (5A2), yellowish orange to purplish gray (6D5, 12D/E3) virgate, slightly reddening when cut (especially near the base). Hymenophore tubulose, adnexed, slightly depressed around stipe; tubes easily separable, pale orange (5A2), 10-12 mm long halfway to the margin; pores at first dark violet brown (11F4) becoming brown (8F5-6). Spore print not recorded.

Macrochemical reactions: $\mathrm{KOH}$ : yellowish brown on pileus and stipe; $\mathrm{NH}_{4} \mathrm{OH}$ : yellowish brown with greenish to pinkish aura on pileus; orange with blue green aura on stipe.

Basidiospores from the type (immature) (5.8-) 6.6-7.5-8.4 $(-8.8) \times(2.9-) 3.2-3.7-4.2(-4.4) \mu \mathrm{m}, Q=(1.6-)$ 1.7-2.04$2.41(-2.47), N=55$, oblong to ellipsoid with suprahilar depression, thin-walled, smooth, yellowish to brownish hyaline in water, yellowish hyaline in $\mathrm{KOH}$ or $\mathrm{NH}_{4} \mathrm{OH}$, inamyloid. From voucher TWO1171 (9-) 9.5-11-13.6 (-14) × (3.9-) 3.9-4.4$4.9(-5.2) \mu \mathrm{m}, N=50 \mathrm{Q}=(2.06-)$ 2.18-2.51-2.98 (-3.11), narrowly ellipsoid to subcylindrical with suprahilar depression, thin-walled, smooth, yellowish to brownish hyaline in water, yellowish hyaline in $\mathrm{KOH}$ or $\mathrm{NH}_{4} \mathrm{OH}$, inamyloid. Basidia 4spored, rarely 2-spored (24-) 25-28-34 (-36) × (8-) 8-9-11 $(-13) \mu \mathrm{m}$, with sterigmata up to $4.5 \mu \mathrm{m}$ long, narrowly clavate to clavate, hyaline, inamyloid. Cheilocystidia (20-) 22-30-39 (43) $\times(4-)$ 4-7-11 (-11) $\mu \mathrm{m}$, frequent, narrowly fusiform to fusiform, thin-walled, hyaline. Pleurocystidia (29-) 29-34-40 ($40) \times(5-)$ 5-6-7 (-7) $\mu \mathrm{m}$, infrequent to rare, same shape as the cheilocystidia, thin-walled, hyaline. H. trama divergent 45$78 \mu \mathrm{m}$ wide, with subregular mediostratum 18-26 $\mu \mathrm{m}$ wide. Pileipellis a palisadoderm when young becoming trichoderm, 97$128 \mu \mathrm{m}$ thick, composed of thin-walled hyphae, with terminal cells $16-47 \times 3-7.5 \mu \mathrm{m}$, cylindrical to subcylindrical with rounded to subacute apex somewhere, slightly yellowish to reddish pale brown in water, mostly hyaline to yellowish pale brown somewhere in $\mathrm{KOH}$ or $\mathrm{NH}_{4} \mathrm{OH}$. Pileus context made of strongly interwoven hyaline hyphae, 6-16.5 $\mu \mathrm{m}$ wide, with scattered loose crystals. Stipitipellis a disrupted hymeniderm, 80-110 $\mu \mathrm{m}$ thick, composed of parallel hyphae, with terminal cell 13-43 × 5-9.5 $\mu \mathrm{m}$ of thin-walled, hyaline hyphae, giving rise to clusters of caulocystidia, basidiole-like cells and scarce basidia, with scattered encrustations. Caulocystidia (24-) 2535-51 (-55) × (7-) 8-10-14 (-14) $\mu \mathrm{m}$, frequent in groups, fusiform, thin-walled, hyaline. Stipe context composed of parallel 4- to 13.5- $\mu \mathrm{m}$-wide hyphae, with scattered encrustations. Clamp connections not seen in any tissue.

Habitat: Solitary to gregarious, on soil, in hill evergreen forest dominated by Castanopsis spp., Lithocarpus spp. mixed with Sh. obtusa, Sh. siamensis, D. obtusifolius and D. costatus.

Distribution: Chiang Mai Province, Northern Thailand.

Additional collections examined: THAILAND: Chiang Mai Province: Mae Taeng District, Behind Wat Pah Deng, 

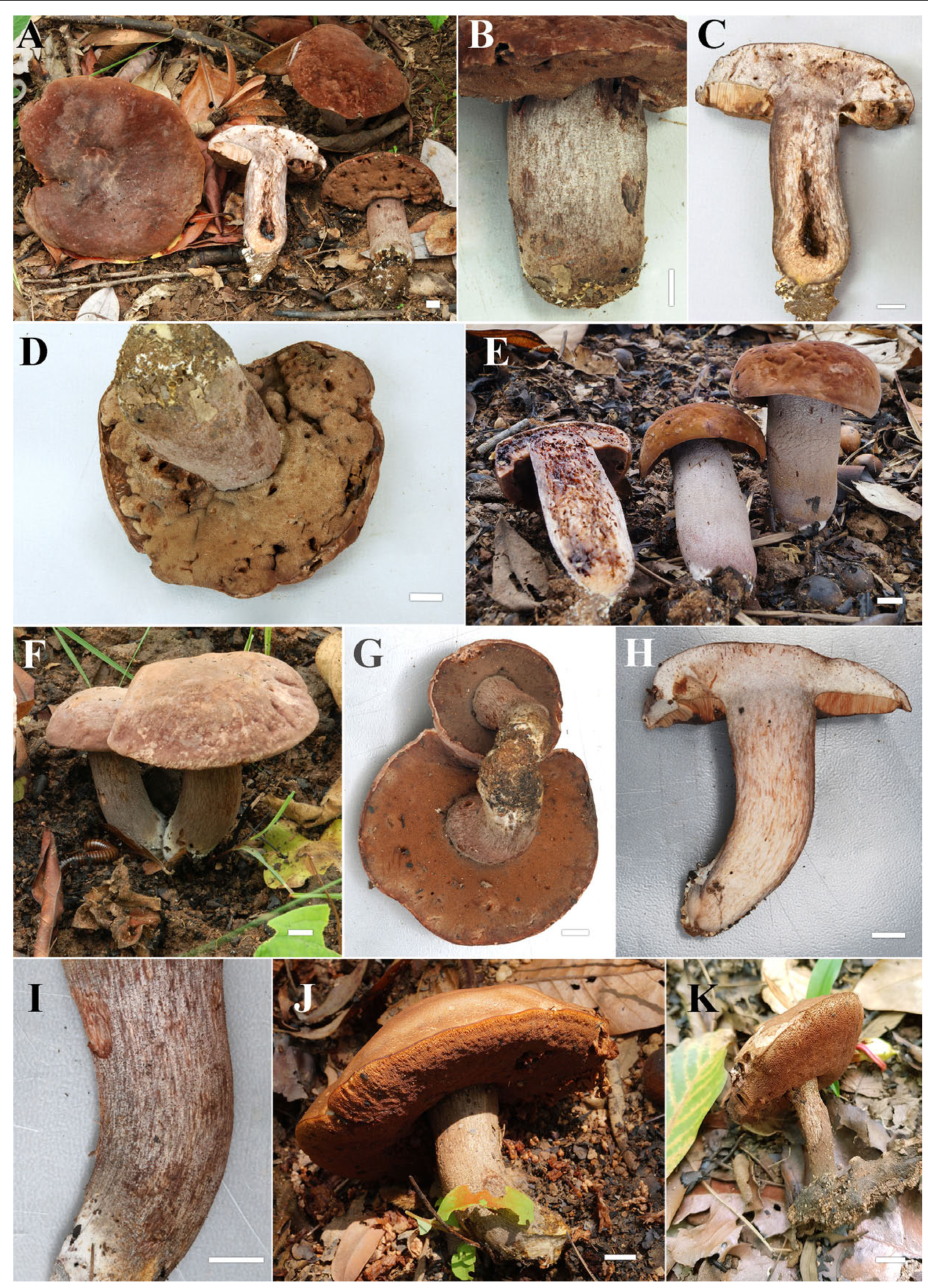

FIGURE 7 | (A-E) Fresh basidiomata of Sutorius pachypus (A-D: OR0411, E: SV0098). (F-K) Fresh basidiomata of Sutorius pseudotylopilus (F-I: OR0378B, J: SV0401, K: SV0415). Scale bars: (A-K) = $1 \mathrm{~cm}$.

$19^{\circ} 06^{\prime} 31^{\prime \prime} \mathrm{N}-98^{\circ} 44^{\prime} 28^{\prime \prime}$ E, elev. 1,070 m, July 7, 2015, Santhiti Vadthanarat, SV0098 (CMUB, BR). -ibid., Baan Mae Sae,

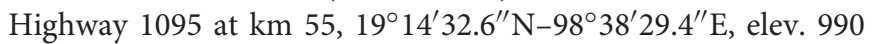
m, June 10, 2006, Osmundson, TWO1171 (MFLU, NY).

Notes: S. pachypus is characterized by the following combination of characteristics: medium-sized basidiomata; wide cylindrical stipe with granulose squamules on the stipe surface that mostly are pale violet gray to violet brown at places; pileipellis is a palisadoderm to trichoderm, subcylindrical terminal cells with rounded to subacute apex; presence of cheilocystidia and pleurocystidia. The holotype (OR0411) has oblong to ellipsoid basidiospores because it is immature. However, a mature specimen (TWO1171) has the typical shape of basidiospores, which are narrowly ellipsoid to subcylindrical with suprahilar depression. The specimen (TWO1171) was originally morphologically identified as S. eximius (Halling et al., 2012), 


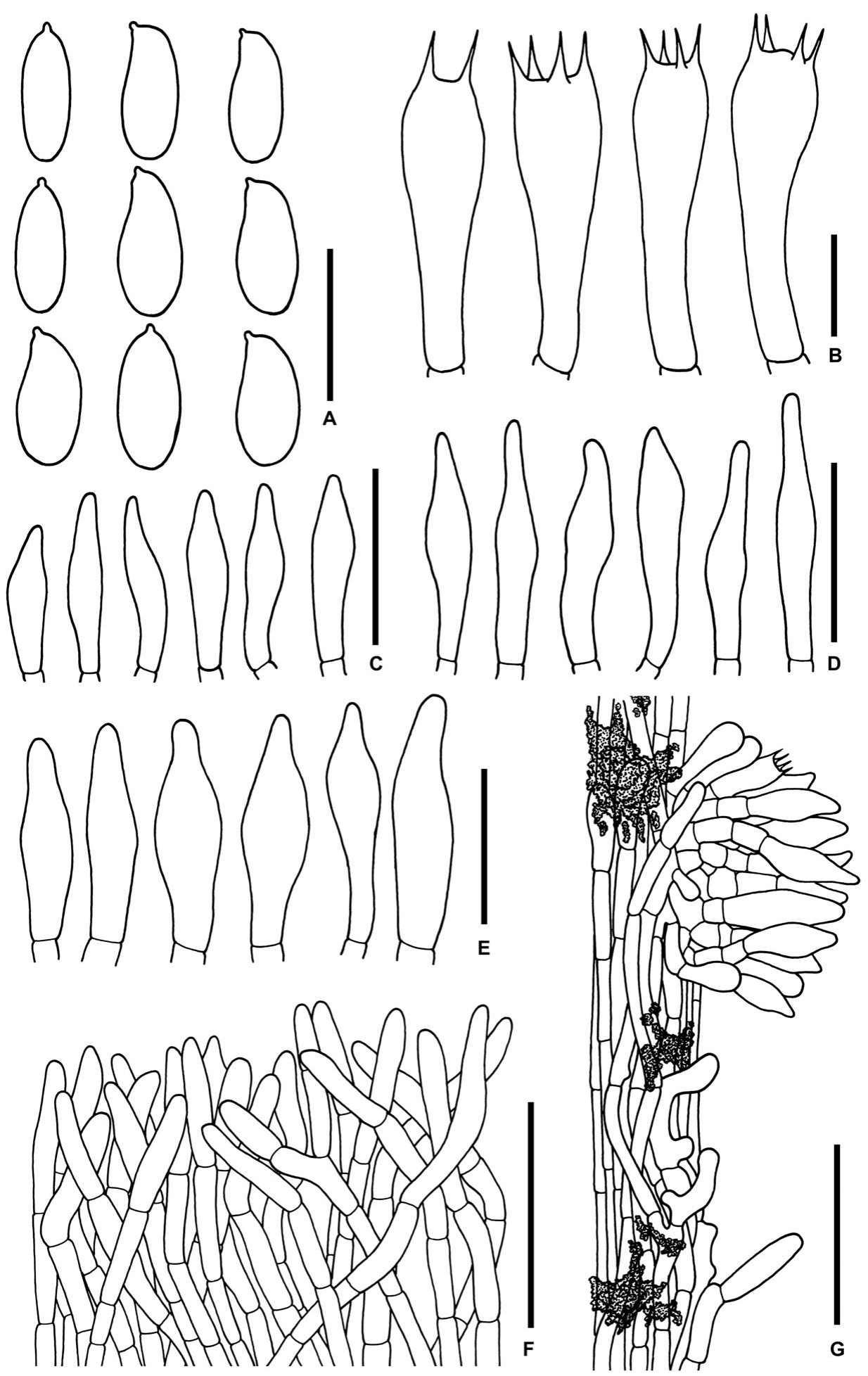

FIGURE 8 | Microscopic features of Sutorius pachypus. (A) Basidiospores. (B) Basidia. (C) Cheilocystidia. (D) Pleurocystidia. (E) Caulocystidia. (F) Pileipellis. (G) Stipitipellis. All drawings were made from OR0411. Scale bars: $(\mathbf{A}, \mathbf{B})=10 \mu \mathrm{m} ;(\mathbf{C}-\mathbf{E})=25 \mu \mathrm{m} ;(\mathbf{F}, \mathbf{G})=50 \mu \mathrm{m}$.

but our more detailed examination and phylogenetic analyses indicate a new species, S. pachypus. Phylogenetically, S. pachypus formed a clade (clade 10) closely related to S. rubinus (clade 9),
S. australiensis (clade 11), and S. mucosus (clade 12). However, all of those differ from S. pachypus mainly in having a narrower stipe with darker granulose squamules. The other differences are as 
follows: S. rubinus has redder basidiomata, lacks pleurocystidia, and has a trichoderm pileipellis with fusiform to broadly fusiform terminal cells with acuminate apex. S. australiensis has darker violet-brown pores (when young), and it has been found so far only in Australia. S. mucosus lacks both cheilocystidia and pleurocystidia and has a waxy to subviscid pileus, an ixotrichoderm pileipellis with fusiform to utriform terminal cells with rounded to tapering apex.

Sutorius pseudotylopilus Vadthanarat, Raspé and Lumyong sp. nov.

MycoBank: MB838061

Figures 7F-K, 9

Typification: THAILAND: Chiang Mai Province: Mae On District, Ban Huay Keaw community forest, $18^{\circ} 51^{\prime} 55^{\prime \prime} \mathrm{N}-$ $98^{\circ} 17^{\prime} 23^{\prime \prime}$ E, elev. 710 m, June 4, 2012, Olivier Raspé and Komsit Wisitrassameewong, OR0378B (MFLU: holotype; BR: isotype).

Etymology: From Greek pseudo-, false, and Tylopilus refers to the macromorphological similarity to the genus Tylopilus.

Description: Basidiomata medium-sized. Pileus $5.0-12 \mathrm{~cm}$ in diameter, at first convex with inflexed margin becoming planoconvex to depressed with straight margin, margin slightly exceeding (1-2 mm); surface even to subrugulose, dull, matted tomentose to cracked tomentose with age, patchy, grayish brown to reddish brown, brown (9-10E3-4, 7F5-6, 8F3-4). Pileus context 10-20 mm thick halfway to the margin, yellowish white to orange white (4A2 to 5A2), with scattered small groups of reddish brown (9F6-7) encrustations, slowly slightly reddening when cut. Stipe central, terete, cylindrical to slightly tapering downward, 7.5-9.5 × 1.5-2.5 cm; surface even, dull, dry, grayish brown to reddish brown (7-8 D/E 3-4), with grayish brown to dark brown (8-9F3-4) fine scabrous squamules; basal tomentum little developed, white to yellowish white. Stipe context solid, orange white (5A2) near the cap to yellowish white (4A2) to greenish white (3B3) at the base, grayish brown to reddish brown (7-8 D/E 3-4) virgate, with scattered brownish gray (8-10E2) or reddish brown (9D4, 9F4-5) encrustations, slowly slightly reddening when cut. Hymenophore tubulose, narrowly adnexed to adnate, subventricose to ventricose, sometimes slightly depressed around the stipe; tubes easily separable, brown to reddish brown (7-8E4-5), 7-15 $\mathrm{mm}$ long halfway to the margin, unchanged or slightly reddening when cut; pores $0.4-$ $0.8 \mathrm{~mm}$ wide at midradius, mostly roundish, at first dark purple (11F3-4) becoming dark brow to brown (8-9F5-6 to 7F5-6) with age. Odor not observed taste slightly acid. Spore print not observed.

Macrochemical reactions: $\mathrm{KOH}$ : yellowish then brownish on the cap, stipe, and pileus context; yellowish or greenish on stipe context; yellowish on hymenium. $\mathrm{NH}_{4} \mathrm{OH}$ : yellowish red on the pileus; yellowish to brownish on pileus context, stipe, stipe context, and hymenium.

Basidiospores [168/3/3] (9.3-) 10.2-11.9-14 (-15.5) $\times(3.4-)$ $3.9-4.3-5(-5) \mu \mathrm{m}, Q=(2.18-)$ 2.4-2.78-3.24 (-3.82). From the type (9.3-) 9.6-11.1-13.1 (-14.1) × (3.4-) 3.8-4.2-4.5 (-4.7) $\mu \mathrm{m}$, $Q=(2.18-)$ 2.25-2.66-3.44 (-3.82), $N=53$, narrowly ellipsoid to subcylindrical with slight of suprahilar depression, thin-walled, smooth, brownish to yellowish hyaline in water, yellowish hyaline in $\mathrm{KOH}$ or $\mathrm{NH}_{4} \mathrm{OH}$, inamyloid. Basidia 4-spored, (19-) 20-24$28(-28) \times(9-) 9-10-11(-11) \mu \mathrm{m}$, with sterigmata up to $4 \mu \mathrm{m}$ long, clavate, hyaline, inamyloid. Cheilocystidia (21-) 21-24-33 $(-33) \times(4-) 4-6-7(-7) \mu \mathrm{m}$, frequent, mostly cylindrical to fusiform with rounded to tapering apex, thin-walled, hyaline. Pleurocystidia not seen. H. trama divergent to boletoid 30$44 \mu \mathrm{m}$ wide, with regular to subregular mediostratum 7.5$23 \mu \mathrm{m}$ wide. Pileipellis an intricate to flattened trichoderm, 85-130 $\mu \mathrm{m}$ thick, made of loosely to moderately interwoven hyphae, thin-walled, mostly hyaline to yellowish pale brown in $\mathrm{KOH}$ or $\mathrm{NH}_{4} \mathrm{OH}$, with slightly curly cylindrical terminal cells 14-46 × 3.5-6 $\mu \mathrm{m}$ with rounded apex, with scattered loose crystals. Pileus context made of strongly interwoven hyaline hyphae, 5.5-8.5 $\mu \mathrm{m}$ wide, with scattered loose crystals. Stipitipellis a disrupted palisadoderm, 90-110 $\mu \mathrm{m}$ thick, with clusters of basidiole-like cells 9-25 $\times 4-9 \mu \mathrm{m}$ of thin-walled, mostly hyaline to pale yellowish brown hyphae, subtended by short chains of 3-4 (sub) isodiametric cells, with scattered encrustations. Caulocystidia (18-) 18-25-30 (-30) × (7-) 7-10$12(-12) \mu \mathrm{m}$, infrequent, fusiform to broadly fusiform, thinwalled, hyaline. Stipe context composed of parallel, 4- to $11-\mu \mathrm{m}$ wide hyphae, with scattered encrustations. Clamp connections not seen in any tissue.

Habitat: Solitary or fasciculate on soil, in dipterocarp forest dominated by D. obtusifolius, D. tuberculatus, Sh. obtusa, and Sh. siamensis and a few Hopea sp., as well as Castanopsis spp.

Distribution: Chiang Mai Province, Northern Thailand.

Notes: Sutorius pseudotylopilus is characterized by the following combination of characteristics: medium-sized basidiomata, purplish to reddish brown basidiomata when young; lack of pleurocystidia; pileipellis constituted by an intricate to flattened trichoderm with cylindrical, slightly curly terminal cells with rounded apex. Phylogenetically, the three S. pseudotylopilus specimens formed a clade (clade 4) sister to three undescribed Sutorius species: clade 1 contains three specimens from China (HKAS59657, HKAS52672, and HKAS56291); Sutorius clade 2 contains another specimen from China (HKAS56291), and Sutorius clade 3 contains one specimen (TWO986) from Costa Rica in Central America. The closest described species is $S$. eximius (American species) in clade 5. However, $S$. eximius morphologically differs from $S$. pseudotylopilus by the following characteristics: stipe context unchanging when injured; presence of pleurocystidia (Singer, 1947; Smith and Thiers, 1971; Halling et al., 2012). Moreover, based on our latest phylogenetics analyses with more Sutorius exemplars, we hypothesize that $S$. eximius is restricted to North America (clade 5).

Additional collections examined: THAILAND: Chiang Mai Province: Mae On District, Ban Huay Kaew community forest, $18^{\circ} 51^{\prime} 56^{\prime \prime} \mathrm{N}-99^{\circ} 17^{\prime} 24^{\prime \prime} \mathrm{E}$, elev. $700 \mathrm{~m}$, June 1, 2017, Santhiti Vadthanarat, SV0401 (CMUB, BR); -ibid., $18^{\circ} 51^{\prime} 55^{\prime \prime} \mathrm{N}-$ $99^{\circ} 17^{\prime} 24^{\prime \prime}$ E, elev. 700 m, May 14, 2018, Santhiti Vadthanarat, SV0415 (CMUB, BR).

Sutorius rubinus Vadthanarat, Raspé and Lumyong sp. nov.
MycoBank: MB838062
Figures 10A-F, 11 

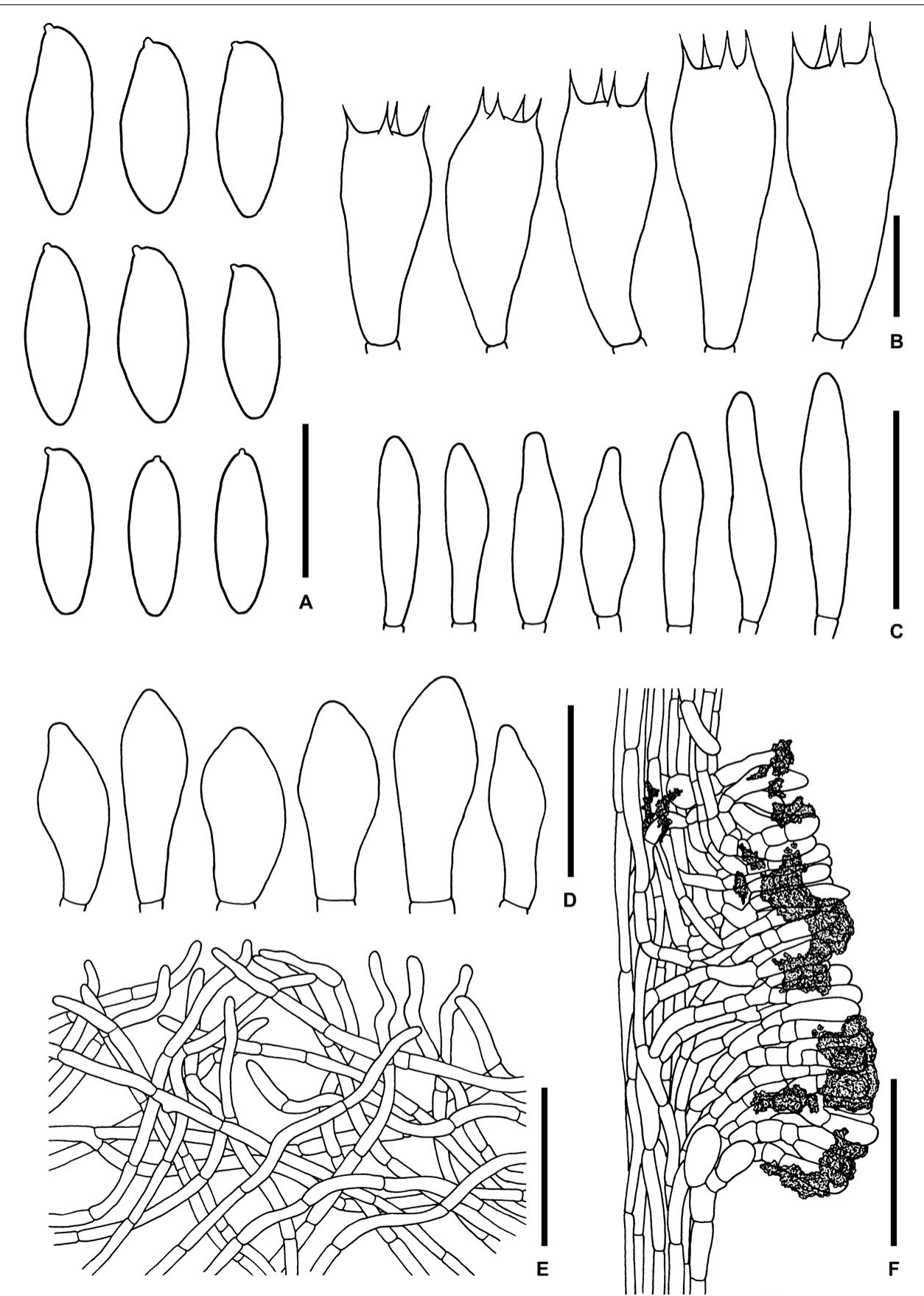

FIGURE 9 | Microscopic features of Sutorius pseudotylopilus. (A) Basidiospores. (B) Basidia. (C) Cheilocystidia. (D) Caulocystidia. (E) Pileipellis. (F) Stipitipellis. All drawings were made from OR0378B. Scale bars: $\mathbf{( A , B )}=10 \mu \mathrm{m} ; \mathbf{( C , D )}=25 \mu \mathrm{m} ; \mathbf{( E , F )}=50 \mu \mathrm{m}$.

Typification: THAILAND: Chiang Mai Province: Mae Taeng District, Pha Deng village, $19^{\circ} 06^{\prime} 38^{\prime \prime} \mathrm{N}-98^{\circ} 44^{\prime} 32^{\prime \prime} \mathrm{E}$, elev. 1,055 m, June 7, 2012, Olivier Raspé and Komsit Wisitrassameewong, OR0403 (MFLU: holotype; BR: isotype).

Etymology: From Latin rubinus, red, referring to basidiomata color.

Description: Basidiomata medium-sized. Pileus $4.5-7.5 \mathrm{~cm}$ in diameter, convex to planoconvex, with slightly deflexed, becoming straight margin, which is slightly exceeding (1-2 mm) especially when young; surface at first subrugulose to rugose becoming scabrous to subrugulose at center, tomentose, reddish dark brown (9F4-6) becoming reddish dark brown (8F5-8) to reddish brown (8D/E6-8) with age, gradually paler to the margin. Pileus context 5-10 mm thick halfway to the margin, orange white (5A2), with scattered small groups of reddish brown (89D4-5) encrustations. Stipe central, terete to slightly compressed, 

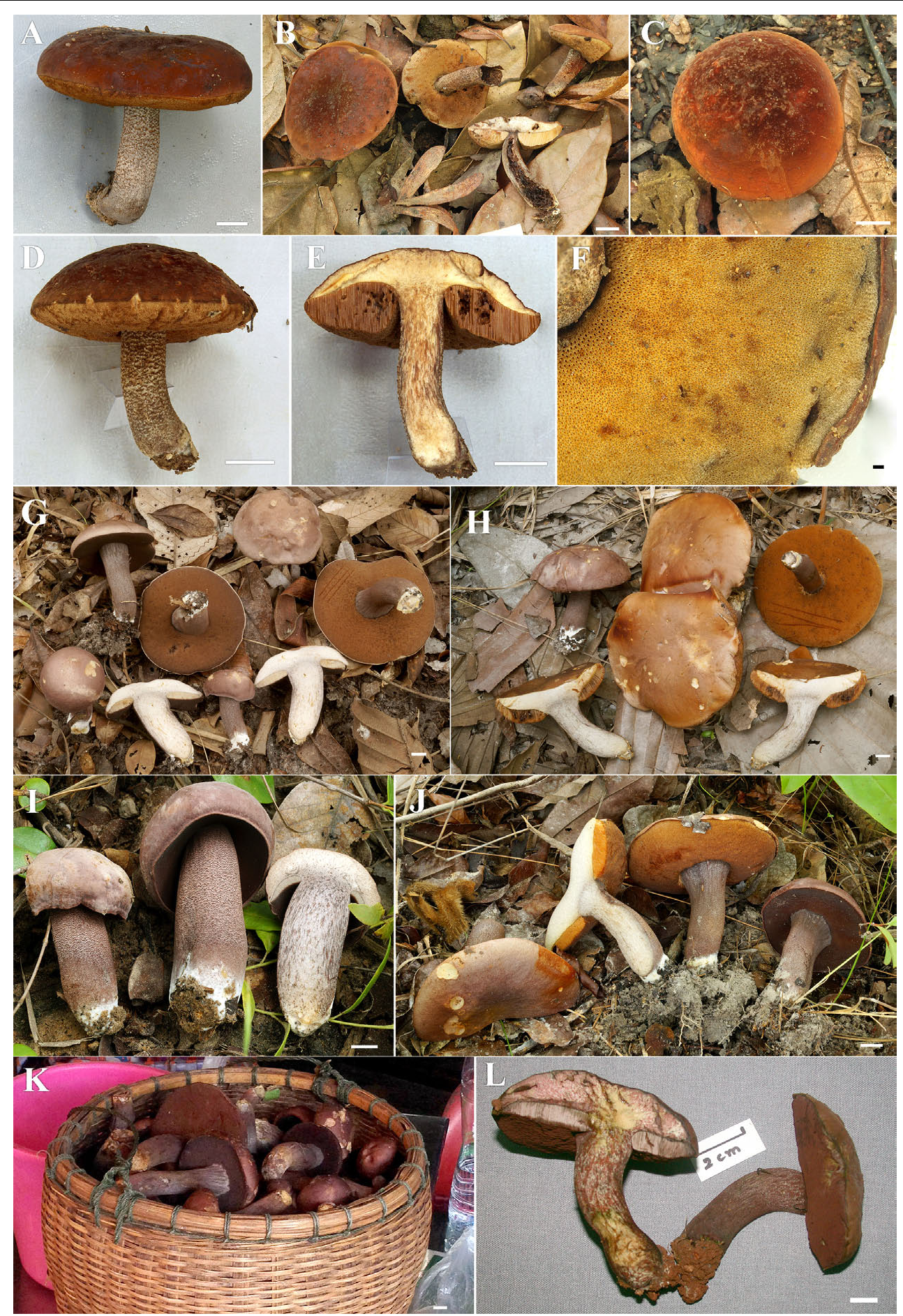

FIGURE 10 | (A-F) Fresh basidiomata of Sutorius rubinus (A: OR0379, B: OR0403, C-E: OR0409, F: pores surface when young in OR1255). (G-K) Fresh basidiomata of Sutorius ubonensis. (G: SV0029, H: SV0032, I: SV0203, J: SV0313, K: SV0353 a collection from a local market in Ubon Ratchathani Province). (L) Fresh basidiomata of Sutorius vellingae specimen voucher ECV3603. Scale bar: (A-L) $=1 \mathrm{~cm}$.

cylindrical to subclavate or tapering downwards, sometimes with subbulbous base, $5-6.5 \times 0.8-2.2 \mathrm{~cm}$; surface finely scabrous, reddish gray to grayish brown (8 D/E $2-3)$ densely covered with reddish brown to dark brown ( $8 \mathrm{~F} 4-5)$ granulose squamules; basal tomentum white to off-white. Stipe context solid, orange white to pinkish white (5A2 to $7 \mathrm{~A} 2$ ), reddish to grayish brown to dark brown (8D-F3-5) virgate, with scattered small groups of reddish brown (8-9D4-5) encrustations, unchanged 

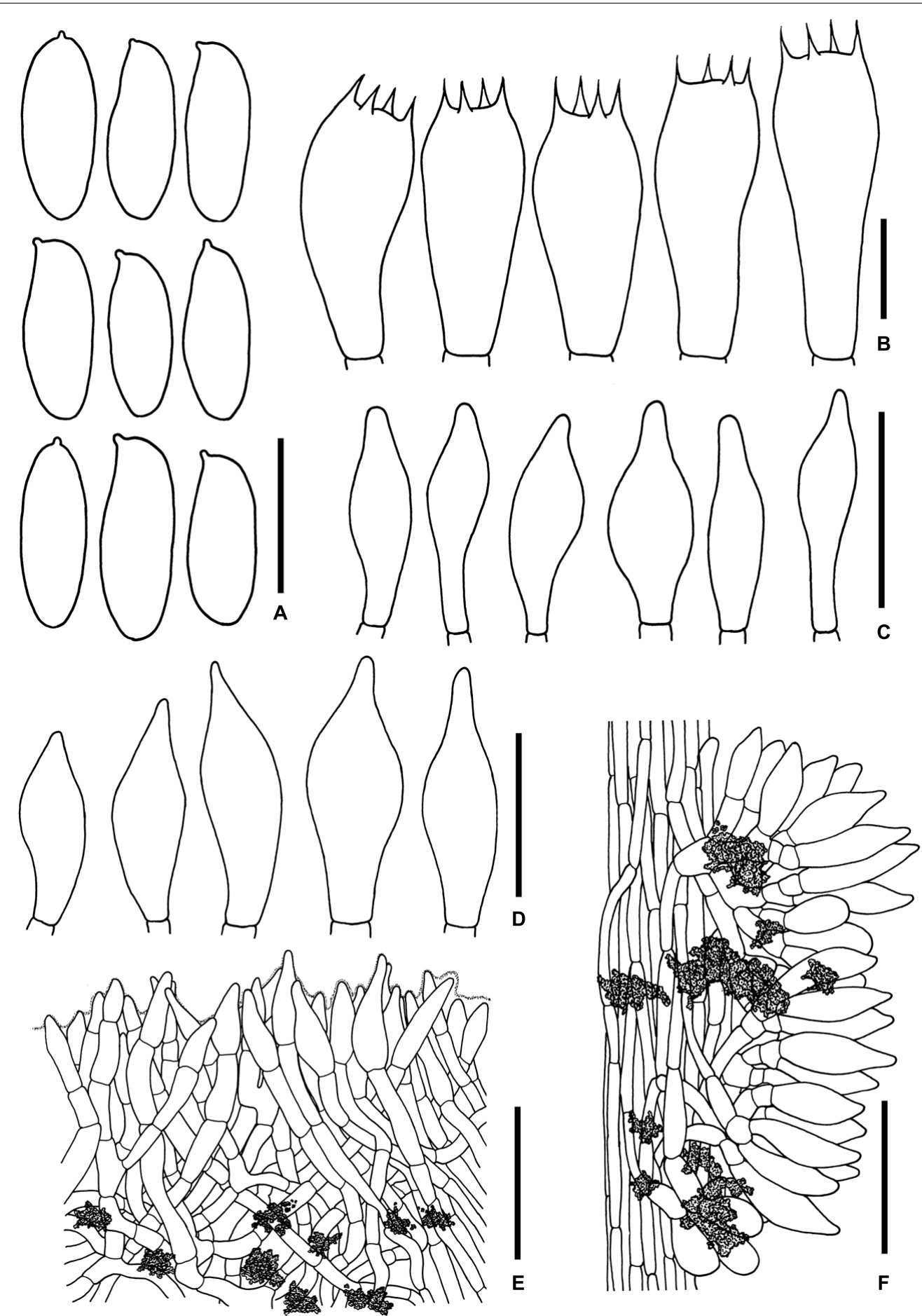

FIGURE 11 | Microscopic features of Sutorius rubinus. (A) Basidiospores. (B) Basidia. (C) Cheilocystidia. (D) Caulocystidia. (E) Pileipellis. (F) Stipitipellis. All drawings were made from OR0403. Scale bars: $(\mathbf{A}, \mathbf{B})=10 \mu \mathrm{m} ; \mathbf{( C , D )}=25 \mu \mathrm{m} ;(\mathbf{E}, \mathbf{F})=50 \mu \mathrm{m}$.

or slightly reddening when cut. Hymenophore tubulose, adnexed to narrowly adnexed, ventricose, slightly depressed around the stipe; tubes dull yellowish white (3A3-4) when young becoming orange-brown (7E7) with age, easily separable, 6$11 \mathrm{~mm}$ long halfway to the margin; pores $0.3-0.5 \mathrm{~mm}$ wide at midradius, mostly roundish, at first grayish orange (5D34) becoming orange to pale orange (5A3-4 to 6B6-7) with age, with scattered small groups of reddish dark brown (8F3-4) encrustations. Odor and taste not observed. Spore print not observed. 
Macrochemical reactions: $\mathrm{KOH}$ : dark brown to black on the stipe, reddish on the hymenophore, negative on the pileus, pileus context, and stipe context.

Basidiospores [168/3/3] (8.7-) 9.6-10.8-12.1 (-13.1) $\times(3.5-)$ 3.9-4.3-4.8 (-5.1) $\mu \mathrm{m}, Q=(2.03-)$ 2.2-2.5-2.82 (-2.96). Form the type (8.7-) 9.3-10.5-12.1 (-12.3) × (3.5-) 3.9-4.4-4.9 (5.1) $\mu \mathrm{m}, Q=(2.03-)$ 2.06-2.4-2.73 (-2.81), $N=57$, narrowly ellipsoid to subcylindrical with slightly suprahilar depression, thin-walled, smooth, yellowish hyaline in $\mathrm{KOH}$ or $\mathrm{NH}_{4} \mathrm{OH}$, inamyloid. Basidia 4-spored (23-) 23-27-31 (-31) × (10-) 1011-13 (-13) $\mu \mathrm{m}$, with sterigmata up to $4 \mu \mathrm{m}$ long, clavate, hyaline, inamyloid. Cheilocystidia (20-) 20-26-32 (-32) $\times(5-)$ 5-7-8 (-8) $\mu \mathrm{m}$, frequent, narrowly fusiform to fusiform with subacute apex, thin-walled, hyaline. Pleurocystidia not seen. $H$. trama divergent, $42-80 \mu \mathrm{m}$ wide, with regular to subregular mediostratum 8-27 $\mu \mathrm{m}$ wide. Pileipellis an intricate trichoderm to slightly gelatinized intricate trichoderm, 60-110 $\mu \mathrm{m}$ thick, made of densely interwoven, thin-walled, smooth, hyaline hyphae 4-12 $\mu \mathrm{m}$ wide, with terminal cells $11-40 \times 5-12 \mu \mathrm{m}$, fusiform to broadly fusiform with acuminate apex, slightly yellowish to reddish pale brown in water, mostly hyaline to yellowish pale brown in $\mathrm{KOH}$ or $\mathrm{NH}_{4} \mathrm{OH}$. Pileus context made of strongly interwoven hyphae, 6-18 $\mu \mathrm{m}$ wide, thinwalled, with scattered loose crystals. Stipitipellis a disrupted hymeniderm, 90-120 $\mu \mathrm{m}$ thick, composed of parallel hyphae, thin-walled, with terminal cells 19-30 × 11-14 $\mu \mathrm{m}$, giving rise to clusters of caulocystidia and basidiole-like cells, with scattered encrustations. Caulocystidia (23-) 23-31-40 (-40) $\times(8-)$ 8-12$18(-18) \mu \mathrm{m}$, frequent, fusiform with subacute apex, thin-walled, hyaline. Stipe context composed of parallel, 8- to $12-\mu \mathrm{m}$-wide hyphae, with scattered encrustations. Clamp connections not seen in any tissue.

Habitat: Solitary to gregarious, on soil, in evergreen hill forest dominated by Castanopsis spp., Lithocarpus spp. mixed with $D$. tuberculatus, D. obtusifolius, Sh. obtusa, and Sh. siamensis.

Distribution: Chiang Mai Province, Northern Thailand

Notes: S. rubinus is characterized by the following combination of characteristics: medium-sized basidiomata, dark reddish brown to reddish brown; lack of pleurocystidia; pileipellis a trichoderm with fusiform to broadly fusiform terminal cells with acuminate apex.

Phylogenetically, all S. rubinus specimens formed a clade (clade 9) close to three Sutorius species including S. pachypus (clade 10), S. australiensis (clade 11), and S. mucosus (clade 12). Nevertheless, they are morphologically different from $S$. rubinus as follows: S. pachypus has on average wider (2.5$4 \mathrm{~cm}$ wide) stipe and has pleurocystidia, and the pileipellis is a palisadoderm composed of subcylindrical with rounded to subacute apex. S. australiensis has dark violet brown pores when young, a trichoderm pileipellis composed of elongated to cylindrical elements with obtuse apex and is found only in Australia. S. mucosus has a waxy to subviscid pileus with an ixotrichoderm forming the pileipellis composed of gelatinized hyphae, with fusiform to utriform terminal cells with rounded to subacute apex.

Additional collections examined: THAILAND: Chiang Mai Province: Mae On District, Ban Huay Keaw community forest, $18^{\circ} 51^{\prime} 55^{\prime \prime} \mathrm{N}-99^{\circ} 17^{\prime} 23^{\prime \prime} \mathrm{E}$, elev. $710 \mathrm{~m}$, June 4, 2012, Jie Chen and Olivier Raspé, OR0379 (MFLU12-0231); Mae Taeng District, Pha Deng village, $19^{\circ} 06^{\prime} 38^{\prime \prime} \mathrm{N}-98^{\circ} 44^{\prime} 32^{\prime \prime} \mathrm{E}$, elev. $1,055 \mathrm{~m}$, June 7, 2012, Olivier Raspé and Komsit Wisitrassameewong, OR0409 (MFLU12-0261); ibid., June 24, 2016, Olivier Raspé, OR1255 (CMUB, BR).

\section{Sutorius ubonensis Vadthanarat, Raspé and Lumyong sp. nov.} MycoBank: MB838066

\section{Figures 10G-K, 12}

Typification: THAILAND: Ubon Ratchathani Province: Trakan Phuet Phon District, Ban Huay Fai community forest, $15^{\circ} 32^{\prime} 44^{\prime \prime} \mathrm{N}-105^{\circ} 10^{\prime} 15^{\prime \prime} \mathrm{E}$, elev. $160 \mathrm{~m}$, July 11, 2014, Santhiti Vadthanarat, SV0032 (CMUB: holotype; BR: isotype).

Etymology: ubonensis refers to Ubon Ratchathani Province where the species was found.

Description: Basidiomata medium-sized. Pileus (5.5) 8.0$10.5(12) \mathrm{cm}$ in diameter, at first hemispherical to convex with straight to slightly inflexed margin, becoming convex to planoconvex to depressed with straight to slightly reflexed margin, margin slightly exceeding (1-2 mm), elastic; surface even to subrugulose, dull, subviscid when wet, minutely tomentose, at first violet to violet brown (10E4) to dark brown (9F5$6)$, later become reddish gray (10B2) to dull red or brownish gray $(10 \mathrm{C} / \mathrm{D} 3)$ at the center, getting paler to brownish gray to $(8 \mathrm{C} 2)$ near the margin, sometimes patchy of reddish gray (8-9C/E3-4) purplish brown (10F4-5) or brown (6E6). Pileus context 10-18 $\mathrm{mm}$ thick halfway to the margin, yellowish white to orange white (4A2 to 5A2), with scattered small groups of reddish brown (9D4-6) encrustations, unchanged. Stipe central, terete to subcompressed, cylindrical to subclavate or tapering downwards (4.8) 5.2-8.5 × 1.8-3.2 cm; surface even to finely scabrous, slightly venose to rimose near the cap, dull to slightly shiny, dry, purplish gray to brownish gray $(9-10 \mathrm{C} / \mathrm{E} 2)$, scattered of reddish brown to dark brown (9F4-5) granulose squamulose; basal tomentum off-white to yellowish white. Stipe context solid, slightly fibrous, yellowish white (4A2 to 5A2) to slightly purplish gray $(15 B / C 2)$ near the stipitipellis, grayish brown to purplish brown (8-9E/F35) virgate, with scattered brownish gray (8-10E2) or reddish brown (9D4 to 9F4-5) encrustations, unchanged. Hymenophore tubulose, adnate, subventricose to ventricose; tubes easily separable, at first purplish to yellowish gray (8C2 to $6 \mathrm{~B} 3$ to $5 \mathrm{~B} 3-4)$ becoming yellowish orange to brownish orange (5B34 to $6 \mathrm{~B} 3-4)$ with age, $8-13 \mathrm{~mm}$ long halfway to the margin; pores $0.3-0.8 \mathrm{~mm}$ wide at midradius, mostly roundish, slightly elongated near the stipe, subregular especially when young, dark brown to purplish dark brown (9F4-6 to 10F3-5) at first, become brown (7F5-6) to orange brown (6E4-5, 7E5-6) with age. Odor fungoid to slightly acidulate. Taste mild to slightly acid. Spore print brownish orange (7C3) to dark brown (7F67) in mass.

Macrochemical reactions: $\mathrm{KOH}$ : rapidly slightly greenish and then yellow on pileus and stipe; yellowish on pileus context, stipe context, and hymenium. $\mathrm{NH}_{4} \mathrm{OH}$ : yellowish with greenish to purplish aura on the cap and stipe; yellowish on pileus context, stipe context, and hymenium. 


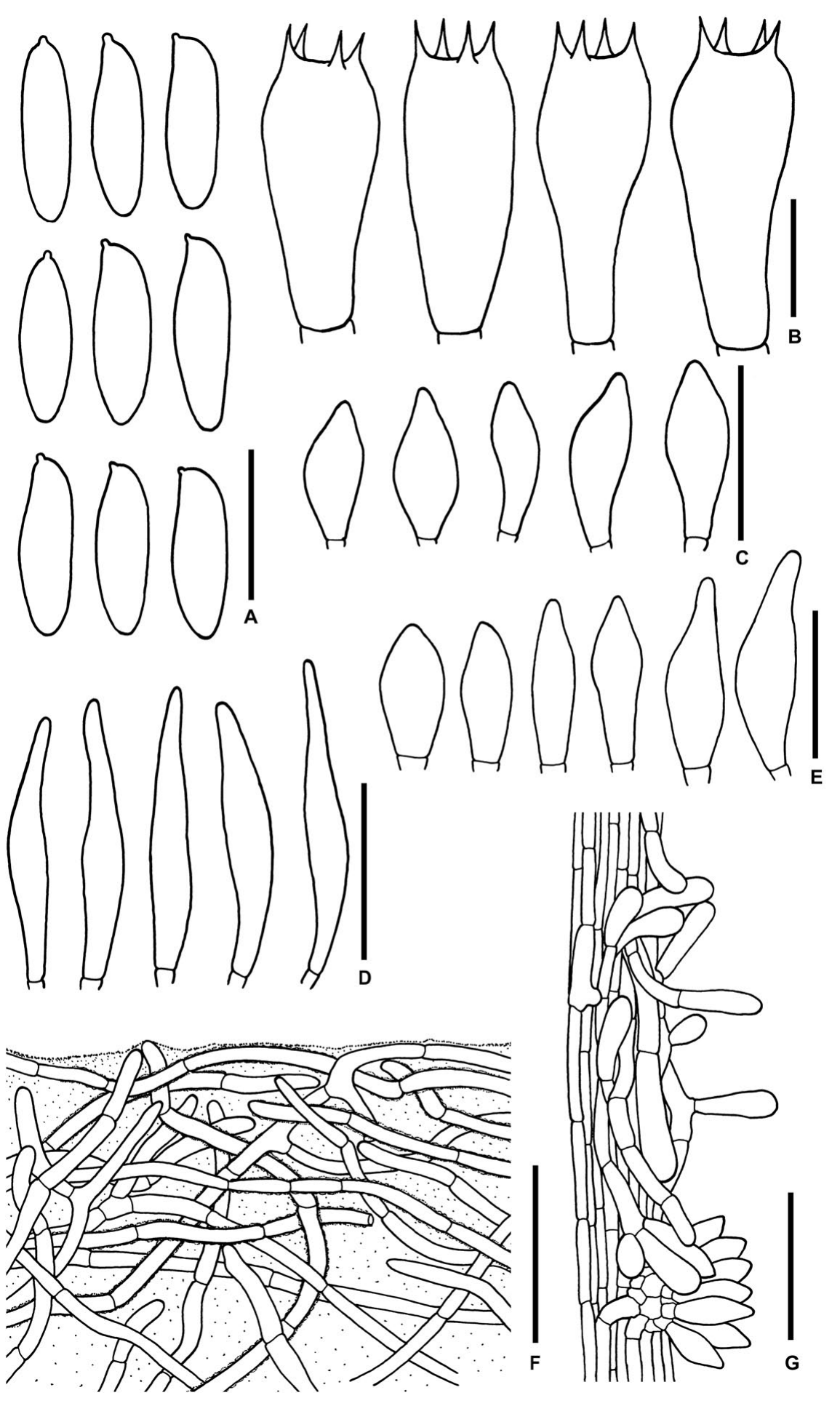

FIGURE 12 | Microscopic features of Sutorius ubonensis. (A) Basidiospores. (B) Basidia. (C) Cheilocystidia. (D) Pleurocystidia. (E) Caulocystidia. (F) Pileipellis. (G) Stipitipellis. Drawings (A-D) were made from SV0032, (F,G) were made from SV0029. Scale bars: $(\mathbf{A}, \mathbf{B})=10 \mu \mathrm{m} ;(\mathbf{C}-\mathbf{E})=25 \mu \mathrm{m} ;(\mathbf{F}, \mathbf{G})=50 \mu \mathrm{m}$.

Basidiospores [172/3/2] (8.7-) 9.8-12-14.7 (-16.8) $\times(3.1-)$ 3.4-4-4.4 (-4.7) $\mu \mathrm{m} Q=(2.61-)$ 2.42-3.01-3.63 (-3.93). From the type (10.1-) 11.1-13.4-16.6 (-16.8) × (3.6-) 3.7-4.1-4.6 (4.7) $\mu \mathrm{m}, Q=(2.68-)$ 2.74-3.27-3.81 (-3.93), $N=57$, narrowly ellipsoid to subcylindrical with slightly suprahilar depression, thin-walled, smooth, brownish to yellowish hyaline in water, yellowish hyaline in $\mathrm{KOH}$ or $\mathrm{NH}_{4} \mathrm{OH}$, inamyloid. Basidia 4spored (19-) 19-22-25 (-25) × (9-) 9-10-11 (-11) $\mu \mathrm{m}$, 
with sterigmata up to $4 \mu \mathrm{m}$ long clavate, hyaline, inamyloid. Cheilocystidia (19-) 19-23-28 (-28) × (6-) 6-8-10 (-10) $\mu \mathrm{m}$, frequent, fusiform, thin-walled, hyaline. Pleurocystidia (34-) 3540-47 (-47) × (5-) 5-6-7 (-7) $\mu \mathrm{m}$, frequent, narrowly fusiform, thin-walled, hyaline. $H$. trama divergent to boletoid, 57-78 $\mu \mathrm{m}$ wide, with 18-42 $\mu \mathrm{m}$ wide of regular mediostratum. Pileipellis a tomentum to slightly gelatinized tomentum, 89-118 $\mu \mathrm{m}$ thick, composed of moderately interwoven, hyaline, thin-walled hyphae, with terminal cells $19-57 \times 5-8.5 \mu \mathrm{m}$, cylindrical with rounded apex, some hyphae were covered with mucus on the wall, at places with scattered of loose crystals. Pileus context made of strongly interwoven hyphae, $8.5-13 \mu \mathrm{m}$ wide, at places with scattered of loose crystals. Stipitipellis hyphae vertically oriented (80-95 $\mu \mathrm{m}$ thick), pointing out of terminal cell $10-35 \times 4-$ $12.5 \mu \mathrm{m}$ of thin-walled with rounded apex, hyaline hyphae, giving rise to clusters of caulocystidia, which (23-) 23-27-35 $(-35) \times(7-) 7-10-13(-13) \mu \mathrm{m}$, frequent in group, fusiform, thin-walled, hyaline, with somewhere scattered of loose crystals especially near the surface. Stipe context composed of parallel, 4.5- to 8 - $\mu \mathrm{m}$-wide hyphae, with scattered small loose crystals. Clamp connections not seen in any tissue.

Habitat: Solitary to gregarious, sometimes fasciculate, on soil in dipterocarp forest dominated by D. obtusifolius, $D$. tuberculatus, Dipterocarpus intricatus, Sh. obtusa, and Sh. siamensis.

Distribution: Ubon Ratchathani Province, Northeastern Thailand.

Notes: S. ubonensis is characterized by the combination of following characteristics: medium sized basidiomata, purplish gray when young becoming purplish to reddish brown with age; unchanged context; pileipellis a tomentum to slightly gelatinized tomentum, with cylindrical terminal elements with rounded apex; found in dipterocarp forest in Northeastern Thailand. Morphologically, S. ubonensis is superficially similar to $S$. eximius in macro-characters especially when young. Both species are also similar in some microscopic characters including the presence and shape of cheilocystidia, pleurocystidia, and caulocystidia, as well as the shape of elements on pileipellis. However, they are different in pileipellis structure, with $S$. eximius having a trichoderm, whereas $S$. ubonensis has a tomentum to slightly gelatinized tomentum. The two species also occur on different continents, with $S$. ubonensis being found in Ubon Ratchathani Province, Northeastern Thailand, Southeast Asia, whereas S. eximius is found in North America (Singer, 1947; Smith and Thiers, 1971; Halling et al., 2012).

Phylogenetically, S. ubonensis (clade 7) was clusters in a poorly supported, long-branch, sister to S. obscuripellis (clade 8) from Chiang Mai Province, Thailand, and S. subrufus (clade 6) from China. However, these two species differ from S. ubonensis as follows: S. obscuripellis has smaller basidiomata and is also paler, especially when young; lacks pleurocystidia; and has a trichoderm pileipellis composed of slightly dark to dark hyphae, with cylindrical terminal cells with subacute to rounded apex. So far, S. obscuripellis has been found only in Chiang Mai Province. S. subrufus also has paler basidiomata; paler pores, especially when young, which are pale brown to brown to pale reddish brown; stipe surface and context turn reddish when injured; a trichoderm pileipellis, composed of rather vertically arranged, with clavate or subclavate terminal cells, with obtuse apex; found in the forests dominated by Fagaceae trees, including Lithocarpus spp. (Chai et al., 2019). So far, S. ubonensis is the only Sutorius species found in Ubon Ratchathani Province, Northeastern Thailand. It occurs in dry dipterocarp forest at the lowest elevation (about 150-175 m) compared to the other Sutorius species.

Additional collections examined: THAILAND: Ubon Ratchathani Province: Trakan Phuet Phon District, Ban Huay Fai community forest, $15^{\circ} 32^{\prime} 43.58^{\prime \prime} \mathrm{N}-105^{\circ} 10^{\prime} 16^{\prime \prime} \mathrm{E}$, elev. 160 m, July 11, 2014, Santhiti Vadthanarat, SV0029 (CMUB, BR); ibid., $15^{\circ} 32^{\prime} 42^{\prime \prime} \mathrm{N}-105^{\circ} 10^{\prime} 16^{\prime \prime} \mathrm{E}$, elev. $175 \mathrm{~m}$, July 24, 2016, Santhiti Vadthanarat, SV0313 (CMUB, BR); -ibid., 15³2'42”N$105^{\circ} 10^{\prime} 16^{\prime \prime} \mathrm{E}$, elev. $160 \mathrm{~m}$, July 15,2017 , Santhiti Vadthanarat,

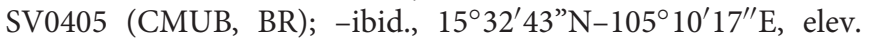
$160 \mathrm{~m}$, July 15, 2017, Santhiti Vadthanarat, SV0407 (CMUB, BR); -ibid., $15^{\circ} 32^{\prime} 43^{\prime \prime} \mathrm{N}-105^{\circ} 10^{\prime} 15^{\prime \prime} \mathrm{E}$, elev. 175 m, July 15, 2017, Santhiti Vadthanarat, SV0410 (CMUB, BR); Sri Suk Village, Khok Tam Lae community forest, $15^{\circ} 35^{\prime} 46^{\prime \prime} \mathrm{N}-105^{\circ} 06^{\prime} 35^{\prime \prime} \mathrm{E}$, elev. 150 m, August 6, 2015, Santhiti Vadthanarat, SV0203 (CMUB, BR); Trakan Phuet Phon market, September 22, 2016, Santhiti Vadthanarat, SV0353 (CMUB, BR).

\section{Sutorius vellingae Vadthanarat, Raspé and Lumyong sp. nov.}

\section{MycoBank: MB838065}

Figures 10L, 13

Typification: THAILAND: Chiang Mai Province: Mae Taeng District, Baan Mae Sae, Highway 1095 at km 55, $19^{\circ} 14^{\prime} 32.6^{\prime \prime} \mathrm{N}-$ 98³8'29.4" E, elev. 990 m, July 4, 2007, Else C. Vellinga, ECV3603 (MFLU: holotype, NY isotype).

Etymology: Named after Else C. Vellinga, who collected the type specimen.

Description: Basidiomata medium-sized. Pileus $7 \times 5.1 \mathrm{~cm}$, convex, margin slightly exceeding (1 $\mathrm{mm})$; surface even to subrugulose, dark brown (8F6-7) with some purple (10F7) shining through at places; pileus context $12-13 \mathrm{~mm}$ thick halfway to the margin, yellowish white (2A2), with scattered small groups of reddish brown (9E/D7-8) encrustations. Stipe central, curved, cylindrical to slightly compressed, $6.8 \times 1.6 \mathrm{~cm}$; surface even to finely scabrous, with some veins on the upper part, dull to slightly shiny, reddish gray to grayish brown (9C2-3), scattered with reddish brown (10F8) granulose squamules to purplish dark brown (9-10F4-6) granulose squamules near the base; basal tomentum little developed, yellowish white; stipe context solid, marmorate to virgate, yellowish white (2-3A2) and brownish red (10E6-7) to violet brown (10F6-7), with scattered small groups of reddish brown (9E/D7-8) encrustations. Hymenophore tubulose, adnate; tubes pinkish white (9A2), $7 \mathrm{~mm}$ long halfway to the margin; pores fine, subregular, violet brown (10F8). Odor fungoid. Taste not distinctive. Spore print not observed.

Basidiospores (9-) 9.5-10.4-11.1 (-11.5) × (3.6-) 3.8-4.2-4.6 (-4.8) $\mu \mathrm{m}, Q=(2.22-)$ 2.22-2.52-2.82 (-2.93), $N=65$, narrowly ellipsoid to subcylindrical with slight suprahilar depression, thinwalled, smooth, brownish to yellowish hyaline in water, yellowish hyaline in $\mathrm{KOH}$ or $\mathrm{NH}_{4} \mathrm{OH}$, inamyloid. Basidia 4-spored (19-) 19-24-28 (-28) × (10-) 10-11-12 (-12) $\mu \mathrm{m}$, with sterigmata 


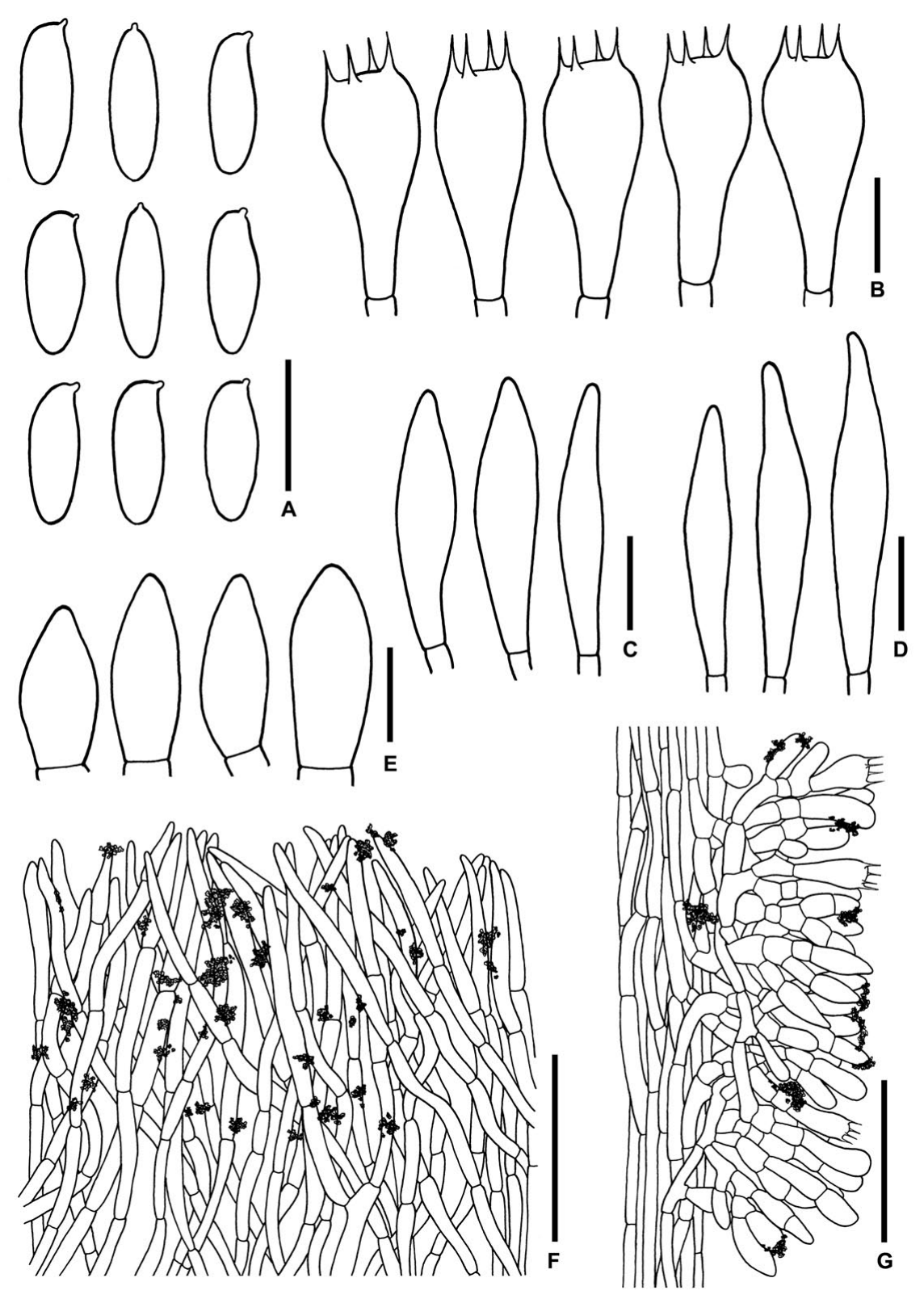

FIGURE 13 | Microscopic features of Sutorius vellingae. (A) Basidiospores. (B) Basidia. (C) Cheilocystidia. (D) Pleurocystidia. (E) Caulocystidia. (F) Pileipellis. (G) Stipitipellis. All drawings were made from ECV3603. Scale bars: $(\mathbf{A}-\mathbf{E})=10 \mu \mathrm{m} ;(\mathbf{F}, \mathbf{G})=50 \mu \mathrm{m}$.

up to $4.5 \mu \mathrm{m}$ long clavate, hyaline, inamyloid. Cheilocystidia $28-$ $29 \times 5-8 \mu \mathrm{m}$, rare, narrowly fusiform to fusiform, thin-walled, hyaline. Pleurocystidia $29-35 \times 6-7 \mu \mathrm{m}$, rare, narrowly fusiform to fusiform, thin-walled, hyaline. $H$. trama divergent, 36-77 $\mu \mathrm{m}$ wide, with 10-31 $\mu \mathrm{m}$ wide of subregular mediostratum. Pileipellis a trichoderm, 110-140 $\mu \mathrm{m}$ thick, thin-walled hyphae, composed of cylindrical terminal cells $23-61 \times 4-7 \mu \mathrm{m}$, with subacute to acute apex, slightly yellowish hyaline in water, slightly greenish to yellowish hyaline in $\mathrm{KOH}$ or $\mathrm{NH}_{4} \mathrm{OH}$, with scattered yellowishbrown parietal encrustations. Pileus context made of moderately interwoven hyaline hyphae, 4-10 $\mu \mathrm{m}$ wide, with scattered reddish brown loose crystals in water or $\mathrm{NH}_{4} \mathrm{OH}$. Stipitipellis a disrupted hymeniderm, 130-175 $\mu \mathrm{m}$ thick, composed of parallel hyaline hyphae anastomosing at places and terminal cells 13$19 \times 7-8 \mu \mathrm{m}$, thin-walled, giving rise to clusters of basidiole-like cells and caulocystidia, with scattered loose crystals. Caulocystidia (17-) 17-21-30 (-30) × (7-) 7-9-11 (-11) $\mu \mathrm{m}$, frequent in groups, fusiform to broadly fusiform, thin-walled, hyaline. Stipe context composed of parallel 4.5 - to $9-\mu \mathrm{m}$-wide hyphae, with scattered loose crystals. Clamp connections not seen in any tissue.

Habitat: Solitary under Castanopsis and Lithocarpus trees.

Distribution: Chiang Mai Province, Northern Thailand.

Notes: The Sutorius specimen voucher ECV3603 was first recognized as a taxon discrete from S. eximius and S. australiensis 
in the original publication of Sutorius, based on phylogenetic analysis of a combined $L S U+$ tef 1 dataset (Halling et al., 2012). However, it was not described as a new species at that time because of the solitary nature of the collection. In this study, that same specimen was further studied and is hypothesized to be a new species, $S$. vellingae. Based on that single collection, $S$. vellingae is characterized by the combination of following characteristics: marked violet brown pore color; pileipellis trichoderm composed of cylindrical terminal cells with subacute to acute apex; rare, narrowly fusiform to fusiform cheilocystidia and pleurocystidia; and its very distinct, isolated phylogenetic position within Sutorius.

\section{Key to Sutorius Species Worldwide}

1 Distribution in the Americas or Australia

$\mathbf{1}^{\prime}$ Distribution in Asia. 2

2 Found in the Americas. S. eximius

2 ' Found in Australia. S. australiensis

3 Pileipellis a palisadoderm or trichoderm to intricate trichoderm.

$3^{\prime}$ Pileipellis a tomentum to slightly gelatinized tomentum composed of cylindrical terminal elements; basidiomata medium sized; at first pileus and pores purple to purplish brown to dark brown becoming purplish to reddish brown...

S. ubonensis

4 Pileipellis composed of wide cylindrical hyphae, with terminal cells fusiform to broadly fusiform or utriform (5-12 $\mu \mathrm{m}$ wide).

5

4 ' Pileipellis composed of cylindrical hyphae, with narrow cylindrical to cylindrical terminal cells (3-7 $\mu$ m wide)........ 7 5 Pileipellis strongly gelatinized, composed of slimy hyphae, with terminal cells fusiform to utriform with rounded to subacute apex

S. mucosus

$5^{\prime}$ Pileipellis not to slightly gelatinized, composed of hyphae with terminal cells fusiform to broadly fusiform with acuminate or tapering apex..

6

6 Basidiomata reddish dark brown to reddish brown; pores grayish orange at first; pileipellis with fusiform to broadly fusiform terminal cells, with acuminate apex........... S. rubinus $\mathbf{6}^{\prime}$ Basidiomata dark lilac to purplish to reddish brown, pores lilac gray at first; pileipellis with fusiform terminal cells, with tapering apex.

S. maculatoides

7 Pileipellis an intricate trichoderm........................................... 8 $7^{\prime}$ Pileipellis a palisadoderm or trichoderm composed of almost vertically arranged hyphae.

9 8 Basidiomata small- to medium-sized, pileus $3.5-6 \mathrm{~cm}$ in diam.; pileipellis composed of cylindrical hyphae, terminal cells cylindrical with subacute to rounded apex, with scattered small parietal encrustations, brown in $\mathrm{KOH}$..... S. obscuripellis $8^{\prime}$ Basidiomata medium-sized, pileus $5-9.5 \mathrm{~cm}$ in diam.; pileipellis composed of cylindrical hyphae with terminal cells cylindrical and slightly curly, mostly hyaline to yellowish pale brown in $\mathrm{KOH}$ S. pseudotylopilus 9 Terminal cells of the pileipellis cylindrical with obtuse to rounded apex; pleurocystidia frequent.. 10 $\mathbf{9}^{\prime}$ Terminal cells of the pileipellis are cylindrical with tapering to acute apex; pleurocystidia rare. S. vellingae
10 Basidiomata with wide cylindrical stipe 5.5-6.5 × 2.5-4 cm; stipe surface unchanging when injured ................ S. pachypus $10^{\prime}$ Basidiomata with subcylindrical stipe 6-10 $\times 1-2.2 \mathrm{~cm}$; stipe surface usually reddening when injured ........ S. subrufus

\section{DISCUSSION}

Sutorius species are morphologically roughly similar to each other by their chocolate to reddish brown or purplish brown basidiomata, with granulose squamules scattered or transversely scissurate scales on the stipe surface. For this reason, macromorphological features are often not enough for species identification. Observation of microscopic characters is needed. The dimensions and $Q$-value of the basidiospores are the characters commonly used to differentiate species in the Boletaceae. In Sutorius, however, the dimensions and $Q$-value of the basidiospores seem to be poor characters for species delineation, because spore size and Q-value, especially the length of the basidiospores, can vary greatly within the same species and sometimes within the same individual. For example, in this study, the basidiospore length of S. ubonensis varied from 10.1 to $16.8 \mu \mathrm{m}$ in the same basidioma, and from 8.7 to $16.8 \mu \mathrm{m}$ between different collections. Therefore, in this study, the dimensions and $Q$-value of basidiospores were not used to differentiate species. On the basis of this study and previously published data, the appropriate set of characters for species identification of Sutorius is the combination of macromorphological features including basidiomata size, color, and stipe surface, and microscopic characters such as the arrangement and shape of terminal cells of the pileipellis, and the presence/absence or frequency of hymenophoral cystidia. Those characters mentioned above show little intraspecific variability and allow a clear-cut species delimitation. Some of the new species, namely, S. mucosus, S. obscuripellis, and S. vellingae, were described based on a single collection or basidiomata. However, all morphological and phylogenetic evidence was reliable and compelling enough to describe those species as new. During our survey in the North of Thailand, starting in 2012, we could not get more collections of those species, which suggests that those species are uncommon or rare. More collections of those species would be useful to better describe and understand the intraspecific genetic and morphological variability.

In the literature, Sutorius eximius has also been reported from Thailand (as T. eximius) (Chantorn et al., 2007; Chandrasrikul et al., 2008; Vadthanarat, 2009; Vadthanarat et al., 2010). Those reports were based on morphological characters only. Our phylogenetic analyses on Thai collections showed that they belong to eight different species, but none of them belongs in S. eximius. The phylogenetically closest species to $S$. eximius (voucher REH9400, from United States) was S. pseudotylopilus, but it differs morphologically and ecologically from $S$. eximius (see in S. pseudotylopilus notes). Morphologically, the most similar species is $S$. ubonensis, which was previously reported as T. eximius from Ubon Ratchathani Province (Vadthanarat, 2009; 
Vadthanarat et al., 2010). Another report of T. eximius was from Nam Nao national park, Northeastern Thailand (Chantorn et al., 2007). We could not obtain the specimen for this study, but it can reasonably be assumed that, again, it belongs to a different species, other than S. eximius.

In Northeastern Thailand, S. ubonensis is one of the wild edible boletes that is collected and consumed by local people. It is called "Hed Pheung Khao Kam" or "Hed Pheung E-Dam," which means purple or blackish bolete. The price of S. ubonensis is usually about 200-300 THB/kg. Local people usually cook it by steaming or boiling over a long time period. They also believe that if it is not properly cooked, it might be poisonous.

In this study, a total of seven new Sutorius species and one new combination are proposed from Thailand and Southeast Asia. Before this study, only three Sutorius species were described worldwide, with one species from each of three continents, the Americas, Australia, and Asia (Halling et al., 2012; Chai et al., 2019). At present, 11 Sutorius species are known including the eight additional species in this study. In addition to those 11 species, our phylogeny (Figure 1) resolved six more terminal clades, which are phylogenetically distinct from all the described species. Among the six species, three are from Africa (Togo, Zimbabwe, and Burundi; clades 14, 15, and 16), two are from Asia (China; clades 1 and 2), and another was from Central America (Costa Rica; clade 3). Moreover, in previous studies, some undescribed Sutorius species were also phylogenetically evidenced from China, Zambia, and Indonesia, but have not yet been described (Halling et al., 2012; Chai et al., 2019). In South America, several Sutorius collections from Guyana were also morphologically identified and reported as T. eximius (Fulgenzi et al., 2007). Unfortunately, those latter collections were not added in our phylogeny because there were no sequences available in GenBank compatible with our phylogenetic study, and we have not been able to obtain the specimens. They might correspond as allies to some of the newly described species in this study. It is noteworthy that, so far, there is not any Sutorius collection known from Europe (Fulgenzi et al., 2007; Halling et al., 2012; Chai et al., 2019). Phylogenetically, the African specimens clustered together in a well-supported subclade within Sutorius, sister to another highly supported subclade comprising American, Australian, and Asian specimens with the exception of $S$. vellingae (ECV3603). S. vellingae (ECV3603) was not in the same clade with the other Asian species, but formed an isolated terminal clade, sister to all other Sutorius species. The pattern of two well-supported clades of African specimens sister to the others was also observed in the genus Pulveroboletus (Badou et al., 2018).

This study has refined the original concept of Sutorius and its members proposed by Halling et al. (2012) and Chai et al. (2019). An unexpectedly large number of Sutorius species were found and described in this study from Northern and Northeastern Thailand. Over a 7-year period (2012-2018), 26 Sutorius collections were obtained, of which 17 were obtained from northern Thailand (seven sites in three districts). These collections belonged to six novel and one known species. The other nine collections were from northeastern Thailand (collected from three sites in one district) and belonged to a single new species, different from those found in northern Thailand. Such a high diversity in a relatively small area, compared the single species known from the Americas and the single species known from Australia, was unexpected. It might be explained, however, by the location of the studied area in the IndoBurma biodiversity hotspot. This hotspot is characterized by a wide variation in land form, climate, and latitude, which has led to the development of diverse natural habitats that support a high biodiversity (van Dijk et al., 2004). Moreover, the complex geological history associated with the Pleistocene climate oscillations has been suggested to produce the many localized centers of endemism observed in the hotspot (van Dijk et al., 2004). Northern Thailand, in particular, has a wide range of elevations, from around $300 \mathrm{~m}$ a.s.l. to $2,565 \mathrm{~m}$ a.s.l. at Doi Inthanon, the highest mountain in Thailand. This results in a diversity of forest types, many of which are dominated by ectomycorrhizal trees belonging to the Dipterocarpaceae, Fagaceae, Betulaceae, or Pinaceae. The center of diversity of Sutorius thus appears to be located in the studied area or possibly more broadly in the Greater Maekong Subregion. This region has been shown to shelter a high diversity of fungi, many of which only recently described or still unknown to science (e.g., Hyde et al., 2018; Thongbai et al., 2018). More studies on Sutorius are, however, needed in order to better document the distribution of this genus and clearly understand its biogeographic structure.

\section{DATA AVAILABILITY STATEMENT}

The datasets presented in this study can be found in online repositories. The names of the repository/repositories and accession number(s) can be found in the article/Supplementary Material.

\section{AUTHOR CONTRIBUTIONS}

OR, SV, and SL conceived and designed the study. SV, OR, and MA analyzed the data. SV, OR, and RH collected the specimens. SV and OR performed the experiments and wrote the manuscript. All authors reviewed and edited the manuscript.

\section{FUNDING}

This research work was partially supported by the Chiang Mai University and the Center of Excellence on Biodiversity (BSC), Office of Higher Education Commission, Thailand (BDC-PG3161005).

\section{ACKNOWLEDGMENTS}

OR is grateful to the National Fund for Scientific Research, Belgium (FNRS) for travel grants. The authors are grateful 
for the permit number 0907.4/4769 granted by the Department of National Parks, Wildlife and Plant Conservation, Ministry of Natural Resources and Environment for collecting in Doi SuthepPui National Park. The publication of this work was financially supported by Mae Fah Luang University.

\section{REFERENCES}

Badou, A. S., De Kesel, A., Raspé, O., Ryberg, M. K., Guelly, A. K., and Yorou, N. S. (2018). Two new African siblings of Pulveroboletus ravenelii (Boletaceae). MycoKeys 43, 115-130. doi: 10.3897/mycokeys.43. 30776

Chai, H., Liang, Z. Q., Xue, R., Jiang, S., Luo, S. H., Wang, Y., et al. (2019). New and noteworthy boletes from subtropical and tropical China. MycoKeys 46, 55-96. doi: $10.3897 /$ mycokeys.46.31470

Chandrasrikul, A., Suwanarit, P., Sangwanit, U., Lumyong, S., Payapanon, A., Sanoamuang, N., et al. (2011). Checklist of Mushrooms (Basidiomycetes) in Thailand. Bangkok: Natural Resources and Environmental Policy and Planning, 432.

Chandrasrikul, A., Suwanarit, P., Sangwanit, U., Morinaga, T., Nishizawa, Y., and Murakami, Y. (2008). Diversity of Mushrooms and Macrofungi in Thailand. Bangkok: Kasersart University Press, 514.

Chantorn, K., Pachinburavan, A., and Sanoamuang, N. (2007). Nine new records of boletes (Boletales, Hymenomycetes) from Nam Nao and Phu Rua national Parks, Thailand. KKU Res. J. 12, 257-264.

Darriba, D., Taboada, G. L., Doallo, R., and Posada, D. (2012). jModelTest2: more models, new heuristics and parallel computing. Nat. Methods 9:772. doi: 10 . 1038/nmeth.2109

Doyle, J. J., and Doyle, J. L. (1990). Isolation of plant DNA from fresh tissue. Focus $12,13-15$.

Fulgenzi, T. D., Henkel, T. W., and Halling, R. E. (2007). Tylopilus orsonianus sp. nov. and Tylopilus eximius from Guyana. Mycologia 99, 622-627. doi: 10.1080/15572536.2007.11832556

Halling, R. E., Nuhn, M., Fechner, N. A., Osmundson, T. W., Soytong, K., Arora, D., et al. (2012). Sutorius: a new genus for Boletus eximius. Mycologia 104, 951-961.

Horak, E. (2011). Revision of Malaysian Species of Boletales s.l. (Basidiomycota) Described by Corner EJH (1972, 1974). Malaysia: Forest Research Institute and Ministry of Natural Resources and Environment, 283.

Hosen, M. I., Feng, B., Wu, G., Zhu, X. T., Li, Y. C., and Yang, Z. L. (2013). Borofutus, a new genus of Boletaceae from tropical Asia: phylogeny, morphology and taxonomy. Fungal Divers. 58, 215-226. doi: 10.1007/s13225012-0211-8

Hyde, K. D., Norphanphoun, C., Chen, J., Dissanayake, A. J., Doilom, M., Hongsanan, S., et al. (2018). Thailand's amazing diversity: up to $96 \%$ of fungi in northern Thailand may be novel. Fungal Divers. 93, 215-239. doi: 10.1007/ s13225-018-0415-7

Katoh, K., and Standley, D. M. (2013). MAFFT Multiple sequence alignment software version 7: improvements in performance and usability. Mol. Biol. Evol. 30, 772-780. doi: 10.1093/molbev/mst010

Kornerup, A., and Wanscher, J. H. (1978). ) Methuen Handbook of Colour, 3rd Edn. London: Eyre Methuen Ltd.

Kuo, M., and Ortiz-Santana, B. (2020). Revision of leccinoid fungi, with emphasis on North American taxa, based on molecular and morphological data. Mycologia 112, 197-211. doi: 10.1080/00275514.2019.168 5351

Li, Y. C., Feng, B., and Yang, Z. L. (2011). Zangia, a new genus of Boletaceae supported by molecular and morphological evidence. Fungal Divers. 49, 125143. doi: 10.1007/s13225-011-0096-y

Li, Y. C., Ortiz-Santana, B., Zeng, N. K., and Feng, B. (2014). Molecular phylogeny and taxonomy of the genus Veloporphyrellus. Mycologia 106, 291-306. doi: 10.3852/106.2.291

Lutzoni, F., Kauff, F., Cox, C. J., McLaughlin, D., Celio, G., Dentinger, B., et al. (2004). Assembling the fungal tree of life: progress, classification, and evolution of subcellular traits. Am. J. Bot. 91, 1446-1480.

\section{SUPPLEMENTARY MATERIAL}

The Supplementary Material for this article can be found online at: https://www.frontiersin.org/articles/10.3389/fmicb. 2021.643505/full\#supplementary-material

Mason-Gamer, R. J., and Kellogg, E. A. (1996). Testing for phylogenetic conflict among molecular data sets in the tribe Triticeae (Gramineae). Syst. Biol. 45, 524-545. doi: 10.1093/sysbio/45.4.524

Matheny, P. B. (2005). Improving phylogenetic inference of mushrooms with RPB1 and RPB2 nucleotide sequences (Inocybe; Agaricales). Mol. Phylogenet. Evol. 35, 1-20. doi: 10.1016/j.ympev.2004.11.014

Miller, M. A., Holder, M. T., Vos, R., Midford, P. E., Liebowitz, T., Chan, L., et al. (2009). The CIPRES portals. CIPRES. Available online at: http://www.phylo.org/ portal2/home (accessed November 8, 2020).

Raspé, O., Vadthanarat, S., De Kesel, A., Degreef, J., Hyde, K. D., and Lumyong, S. (2016). Pulveroboletus fragrans, a new Boletaceae species from Northern Thailand, with a remarkable aromatic odor. Mycol. Prog. 15:38.

Reeb, V., Lutzoni, F., and Roux, C. (2004). Contribution of RPB2 to multilocus phylogenetic studies of the euascomycetes (Pezizomycotina, Fungi) with special emphasis on the lichen-forming Acarosporaceae and evolution of polyspory. Mol. Phylogenet. Evol. 32, 1036-1060. doi: 10.1016/j.ympev.2004.04.012

Rehner, S. A., and Buckley, E. (2005). A Beauveria phylogeny inferred from nuclear ITS and EF1- $\alpha$ sequences: evidence for cryptic diversification and links to Cordyceps teleomorphs. Mycologia 97, 84-98. doi: 10.1080/15572536.2006. 11832842

Ronquist, F., Teslenko, M., van der Mark, P., Ayres, D., Darling, A., Höhna, S., et al. (2012). MrBayes 3.2: efficient bayesian phylogenetic inference and model choice across a large model space. Syst. Biol. 61, 539-542. doi: 10.1093/sysbio/sys029

Singer, R. (1947). The boletoideae of florida with notes on extralimital species III. Am. Midl. Nat. 37, 1-135. doi: 10.2307/2421647

Smith, A. H., and Thiers, H. D. (1971). The Boletes of Michigan. Ann Arbor: University of Michigan Press.

Stamatakis, A. (2006). RAxML-vi-hpc: maximum likelihood-based phylogenetic analyses with thousands of taxa and mixed models. Bioinformatics 22, 26882690. doi: 10.1093/bioinformatics/btl446

Thongbai, B., Hyde, K. D., Lumyong, S., and Raspé, O. (2018). High undescribed diversity of Amanita section Vaginatae in northern Thailand. Mycosphere 9, 462-494. doi: 10.5943/mycosphere/9/3/3

Vadthanarat, S. (2009). Some Ecological and Biological Characters of Brownish Purple Bolete, Tylopilus eximius Peck. Bachelor's thesis, Ubon Ratchathani University, Thailand

Vadthanarat, S., Lumyong, S., and Raspé, O. (2019). Cacaoporus, a new Boletaceae genus, with two new species from Thailand. MycoKeys 54, 1-29. doi: 10.3897/ mycokeys.54.35018

Vadthanarat, S., Raspé, O., and Lumyong, S. (2018). Phylogenetic affinities of the sequestrate genus Rhodactina (Boletaceae), with a new species, $R$. rostratispora from Thailand. MycoKeys 29, 63-80. doi: 10.3897/mycokeys.29.22572

Vadthanarat, S., Tanpong, S., and Pukahuta, C. (2010). "Inventory survey of wild mushrooms in community forests in Trakarn Phuetphon, Ubon Ratchathani," in The Proceedings of the 4th Ubon Ratchathani University Research Conference, (Thailand: Ubon Ratchathani).

van Dijk, P. P., Tordoff, A. W., Fellowes, J., Lau, M., and Jinshuang, M. (2004). "Indo-Burma," in Hotspots Revisited. Earth's Biologically Richest and Most Endangered Terrestrial Ecoregions, eds R. A. Mittermeier, P. G. Gil, and M. Hoffman (Chicago: University of Chicago Press), 323-330.

Wu, G., Feng, B., Xu, J., Zhu, X. T., Li, Y. C., Zeng, N. K., et al. (2014). Molecular phylogenetic analyses redefine seven major clades and reveal 22 new generic clades in the fungal family Boletaceae. Fungal Divers. 69, 93-115. doi: 10.1007/ s13225-014-0283-8

Wu, G., Li, Y. C., Zhu, X. T., Zhao, K., Han, L. H., Cui, Y. Y., et al. (2016). One hundred noteworthy boletes from China. Fungal Divers. 81, 25-188. doi: 10.1007/s13225-016-0375-8

Zhao, K., Wu, G., Feng, B., and Yang, Z. L. (2014). Molecular phylogeny of Caloboletus (Boletaceae) and a new species in East Asia. Mycol. Prog. 13, 1127-1136. 
Zhu, X. T., Wu, G., Zhao, K., Halling, R. E., and Yang, Z. L. (2015). Hourangia, a new genus of Boletaceae to accommodate Xerocomus cheoi and its allied species. Mycol. Prog. 14:37.

Conflict of Interest: The authors declare that the research was conducted in the absence of any commercial or financial relationships that could be construed as a potential conflict of interest.
Copyright (C) 2021 Vadthanarat, Halling, Amalfi, Lumyong and Raspé. This is an open-access article distributed under the terms of the Creative Commons Attribution License (CC BY). The use, distribution or reproduction in other forums is permitted, provided the original author(s) and the copyright owner(s) are credited and that the original publication in this journal is cited, in accordance with accepted academic practice. No use, distribution or reproduction is permitted which does not comply with these terms. 